\title{
Dynamics of a Stochastic Functional System for Wastewater Treatment
}

\author{
Xuehui Ji and Sanling Yuan \\ College of Science, University of Shanghai for Science and Technology, Shanghai 200093, China \\ Correspondence should be addressed to Sanling Yuan; sanling@usst.edu.cn
}

Received 20 January 2014; Accepted 2 February 2014; Published 24 March 2014

Academic Editor: Weiming Wang

Copyright (C) 2014 X. Ji and S. Yuan. This is an open access article distributed under the Creative Commons Attribution License, which permits unrestricted use, distribution, and reproduction in any medium, provided the original work is properly cited.

The dynamics of a delayed stochastic model simulating wastewater treatment process are studied. We assume that there are stochastic fluctuations in the concentrations of the nutrient and microbes around a steady state, and introduce two distributed delays to the model describing, respectively, the times involved in nutrient recycling and the bacterial reproduction response to nutrient uptake. By constructing Lyapunov functionals, sufficient conditions for the stochastic stability of its positive equilibrium are obtained. The combined effects of the stochastic fluctuations and delays are displayed.

\section{Introduction}

In the last few years, the use of mathematical models describing wastewater treatment is gaining attention as a promising method [1-6]. A basic chemostat model describing substrate-microbe interaction in an activated sludge process is as follows:

$$
\begin{gathered}
\frac{d S}{d t}=\frac{Q\left(S^{0}-S\right)}{V}-\frac{k x S}{K_{S}+S} \frac{D_{\mathrm{O}}}{K_{\mathrm{O}}+D_{\mathrm{O}}}, \\
\frac{d x}{d t}=x\left(\frac{k Y S}{K_{S}+S}-K_{d}\right) \frac{D_{\mathrm{O}}}{K_{\mathrm{O}}+D_{\mathrm{O}}}-\frac{Q_{w} x}{V},
\end{gathered}
$$

where $S(t)$ and $x(t)$ represent the concentrations of the substrate (biochemical oxygen demand) and microbes in an aeration tank at time $t$, respectively. $Q$ is the washout rate, $S^{0}$ is the input concentration of the substrate, and $V$ is the effective volume of the aeration tank; $k$ is the maximum uptake rate of the substrate; $K_{S}$ and $K_{\mathrm{O}}$ are the half-saturation constants of the substrate and oxygen; respectively, $K_{d}$ is the decay rate of microbes and $Q_{w}$ is the emission rate of the sludge; $D_{\mathrm{O}}$ is the concentration of the dissolved oxygen and $D_{\mathrm{O}} /\left(K_{\mathrm{O}}+D_{\mathrm{O}}\right)$ is a switching function describing the effect of $D_{\mathrm{O}}$ on the uptake rate $k$ and the decay rate $K_{d} ; Y \in(0,1)$ is the ratio of the concentration of mixed liquor suspended solids to the substrate. Some extensions and generalizations of the model have been proposed by many researchers (see [7-27], etc.).

Even though deterministic model (1) has a stable positive equilibrium $\left(S^{*}, x^{*}\right)$ under certain conditions, oscillations have been observed frequently in the growth of microbes during the experiments $[28,29]$, which have also been confirmed by many mathematical works for some extended chemostat models incorporating factors such as time delay [15-18, 3032], periodic nutrient input [19-21,33-35], feedback control [22-24], and stochastic environmental perturbations [2527]. For a better understanding of microbial population dynamics in the activated sludge process, we take two steps towards developing model (1).

On the one hand, we take into account time delays that may exist in the process of wastewater treatment. By the death regeneration theory of Dold and Marais [36], the active biomass dies at a certain rate; of the biomass lost, the biodegradable portion adds to the slowly biodegradable organic matter which passes through the various stages to be utilised for active biomass synthesis, which requires some time for the completion of the regeneration. Also there is a time delay that accounts for the time lapse between the uptakes of substrates and the incorporation of these substrates, which has ever been observed from chemostat experiments with microalgae Chlamidomonas Reinhardii even when the limiting nutrient is at undetectable small 
concentration (see $[37,38]$, etc.). In the recent years, chemostat models with such time delays have been given much attention (see, e.g., $[9,14,16-18,39]$, etc.). In this paper, we will use distributed delays to describe the nutrient recycling and the time lapse between the uptakes of nutrient and the incorporation of this nutrient with delay kernels $f(s)$ and $g(s)$, respectively.

On the other hand, in a real process of wastewater treatment there will be fluctuations in concentration of the substrate and microbe population due to stochastic perturbations from external sources such as temperature, light, and the like, or inherent sources in the chemical-physical and biological processes [40]. So we assume that model (1) is exposed by stochastic perturbations which are of white noise type and are proportional to the distances $S(t), x(t)$ from values of the positive equilibrium $S^{*}, x^{*}$, influence on the $\dot{S}(t)$ and $\dot{x}(t)$, respectively. By this way, model (1) becomes in the following form:

$$
\begin{aligned}
d S= & {\left[\frac{\mathrm{Q}\left(S^{0}-S\right)}{V}-k U(S) \frac{D_{\mathrm{O}}}{K_{\mathrm{O}}+D_{\mathrm{O}}}\right.} \\
& \left.+\mu K_{d} \frac{D_{\mathrm{O}}}{K_{\mathrm{O}}+D_{\mathrm{O}}} \int_{0}^{\infty} f(s) x(t-s) d s\right] d t \\
& +\sigma_{1}\left(S-S^{*}\right) d B_{1}(t), \\
d x= & {\left[x\left(Y k \int_{0}^{\infty} g(s) U(S(t-s)) d s-K_{d}\right)\right.} \\
& \left.\times \frac{D_{\mathrm{O}}}{K_{\mathrm{O}}+D_{\mathrm{O}}}-\frac{Q_{w} x}{V}\right] d t+\sigma_{2}\left(x-x^{*}\right) d B_{2}(t),
\end{aligned}
$$

where $B_{i}(t)(i=1,2)$ are standard independent Wiener processes and $\sigma_{i} \geq 0(i=1,2)$ represent the intensities of the noises. $\mu \in(0,1)$ is the fraction of the substrate regenerated from the dead biomass; $U(S)$ is a general specific growth function.

Recently, stochastic biological systems and stochastic epidemic models have been studied by many authors; see, for example, Mao et al. [41, 42], Jiang et al. [43, 44], Liu and Wang $[45,46]$, and the references cited therein. But, as far as we know, there are few works on model (2). In this paper, our main purpose is to study the combined effect of the noises and delays on the dynamics of model (2), that is, whether and how the noises and delays affect the stability of $E^{*}$. By the construction of appropriate Lyapunov functionals, we will show that the positive equilibrium keeps stochastically stable if the noises and delays are small. Furthermore, the sensitivities of the stability of $E^{*}$ with respect to the delays and noises are also discussed.

The paper is organized as follows. We first establish some preliminary results in Section 2. By constructing Lyapunov function(al)s, sufficient conditions for the stochastic stability of the positive equilibrium of the model without and with delays are obtained, respectively, in Sections 3 and 4. Numerical simulations and discussions are finally presented in Section 5.

\section{Some Preliminaries}

Define $Q / V=D, Q_{w} / V=D_{w}, k\left(D_{\mathrm{O}} /\left(K_{\mathrm{O}}+D_{\mathrm{O}}\right)\right)=m$, $K_{d}\left(D_{\mathrm{O}} /\left(K_{\mathrm{O}}+D_{\mathrm{O}}\right)\right)=D_{1}$, and $Y=\gamma$. Then model (2) can be simplified as follows:

$$
\begin{aligned}
d S= & {\left[D\left(S^{0}-S\right)-m U(S) x\right.} \\
& \left.+\mu D_{1} \int_{0}^{\infty} f(s) x(t-s) d s\right] d t \\
& +\sigma_{1}\left(S-S^{*}\right) d B_{1}, \\
d x=x & {\left[-\left(D_{w}+D_{1}\right)+\gamma m \int_{0}^{\infty} g(s) U(S(t-s)) d s\right] d t } \\
& +\sigma_{2}\left(x-x^{*}\right) d B_{2}
\end{aligned}
$$

with initial value conditions

$$
\begin{array}{r}
S(\theta, \omega)=\varphi_{1}(\theta) \geq 0, \quad x(\theta, \omega)=\varphi_{2}(\theta) \geq 0, \\
\theta \in(-\infty, 0],
\end{array}
$$

where $\varphi_{1}(\theta), \varphi_{2}(\theta) \in \mathscr{B} \mathscr{C}\left((-\infty, 0], \mathbb{R}_{+}\right)$, the families of bounded continuous functions from $(-\infty, 0]$ to $\mathbb{R}_{+}$.

The corresponding deterministic model of (3) is

$$
\begin{gathered}
\dot{S}=D\left(S^{0}-S\right)-m U(S) x+\mu D_{1} \int_{0}^{\infty} f(s) x(t-s) d s, \\
\dot{x}=-\left(D_{w}+D_{1}\right) x+\gamma m x \int_{0}^{\infty} g(s) U(S(t-s)) d s,
\end{gathered}
$$

the special case of which when $D=D_{w}$ has ever been investigated by $\mathrm{He}$ et al. [18]. It is easy to see that model (5) has a positive equilibrium $E^{*}\left(S^{*}, x^{*}\right)$ provided that

$$
D_{w}+D_{1}<\gamma m, \quad S^{0}>S^{*},
$$

where

$$
S^{*}=U^{-1}\left(\frac{D_{w}+D_{1}}{\gamma m}\right), \quad x^{*}=\frac{D\left(S^{0}-S^{*}\right)}{m U\left(S^{*}\right)-\mu D_{1}} .
$$

$E^{*}\left(S^{*}, x^{*}\right)$ is globally asymptotically stable provided that the average delays are sufficiently small. Obviously, $E^{*}$ is still an equilibrium of stochastic model (3) if condition (6) holds.

We assume that function $U(S)$ is nonnegative satisfying

$$
\begin{gathered}
U(0)=0, \quad U^{\prime}(S)>0 \\
U^{\prime \prime}(S)<0 \quad \text { for } S>0, \quad \lim _{S \rightarrow \infty} U(S)=1 .
\end{gathered}
$$

And we extend the function $U(S)$ by defining

$$
U(S)=U^{\prime}(0) S+\frac{1}{2} U^{\prime \prime}(0) S^{2} \quad \text { for } S \leq 0,
$$

so that $U$ is well defined in $\mathbb{R}$ and is still of class $\mathscr{C}^{2}$ in $\mathbb{R}$. Thus one can write

$$
U(S)=a+b\left(S-S^{*}\right)+F\left(S-S^{*}\right),
$$


where $F$ represents terms of order $\geq 2$ in $S-S^{*}$. Noting also that $a=U\left(S^{*}\right)$ and $b=U^{\prime}\left(S^{*}\right)$, by condition (6), it follows that $m a>\mu D_{1}$.

Introduce new variables $u_{1}=S-S^{*}, u_{2}=x-x^{*}$; then model (3) can be rewritten as follows:

$$
\begin{aligned}
d u_{1}= & {\left[-\left(D+m b x^{*}\right) u_{1}+\mu D_{1} \int_{0}^{\infty} f(s) u_{2}(t-s) d s\right.} \\
& \left.\quad-m a u_{2}+F_{1}\left(u_{1}, u_{2}\right)\right] d t+\sigma_{1} u_{1} d B_{1}, \\
d u_{2}= & {\left[\gamma m b x^{*} \int_{0}^{\infty} g(s) u_{1}(t-s) d s\right.} \\
& \left.+\widetilde{F}_{2}\left(u_{1}, u_{2}\right)\right] d t+\sigma_{2} u_{2} d B_{2},
\end{aligned}
$$

where

$$
\begin{gathered}
F_{1}=-m b u_{1} u_{2}-m F\left(u_{1}\right) u_{2}-m x^{*} F\left(u_{1}\right), \\
\widetilde{F}_{2}=\gamma m b u_{2} \int_{0}^{\infty} g(s) u_{1}(t-s) d s \\
\quad+\left(u_{2}+x^{*}\right) \gamma m \int_{0}^{\infty} g(s) F\left(u_{1}(t-s)\right) d s .
\end{gathered}
$$

Note that if $f(s)=g(s)=\delta(0)$, then model (11) has the form

$$
\begin{gathered}
d u_{1}=\left[-\left(D+m b x^{*}\right) u_{1}+\left(\mu D_{1}-m a\right) u_{2}\right. \\
\left.+F_{1}\left(u_{1}, u_{2}\right)\right] d t+\sigma_{1} u_{1} d B_{1}, \\
d u_{2}=\left[\gamma m b x^{*} u_{1}+F_{2}\left(u_{1}, u_{2}\right)\right] d t+\sigma_{2} u_{2} d B_{2},
\end{gathered}
$$

where

$$
F_{2}=\gamma m b u_{1} u_{2}+\left(u_{2}+x^{*}\right) \gamma m F\left(u_{1}\right) .
$$

Obviously, model (13) has the same equilibrium $(0,0)$ as model (11), and the stochastic stability of the positive equilibrium $E^{*}$ of model (3) is equivalent to the zero solution of model (11). We wonder how the stochastic perturbations and delays affect the dynamics of model (3) or (11).

Before starting our analysis, we first give some basic theories in stochastic differential equations and stochastic functional differential equations [47-49]. Let $\left.\left(\Omega, \mathscr{F}_{,}, \mathscr{F}_{t}\right\}_{t \geq 0}, P\right)$ be a complete probability space with a filtration $\left\{\mathscr{F}_{t}\right\}_{t \geq 0}$ satisfying the usual conditions (i.e., it is right continuous and $\mathscr{F}_{0}$ contains all $P$-null sets $)$. Let $B_{i}(i=1,2, \ldots, n)$ be the Brownian motions defined on this probability space. Consider the following $n$-dimensional stochastic differential equation:

$$
d x(t)=f(x(t), t) d t+g(x(t), t) d B(t), \quad t \geq t_{0} .
$$

Definition 1. The trivial solution of system (15) is said to be as follows:

(i) stochastically stable or stable in probability if for every pair of $\varepsilon \in(0,1)$ and $r>1$, there exists a $\delta=$ $\delta\left(\varepsilon, r, t_{0}\right)>0$ such that

$$
P\left\{\left|x\left(t ; t_{0}, x_{0}\right)\right|<r \forall t \geq t_{0}\right\} \geq 1-\varepsilon,
$$

whenever $\left|x_{0}\right|<\delta$. Otherwise, it is said to be stochastically unstable,

(ii) stochastically asymptotically stable if it is stochastically stable and, moreover, for every $\varepsilon \in(0,1)$, there exists a $\delta_{0}=\delta_{0}\left(\varepsilon, t_{0}\right)>0$ such that

$$
P\left\{\lim _{t \rightarrow \infty} x\left(t ; t_{0}, x_{0}\right)=0\right\} \geq 1-\varepsilon,
$$

whenever $\left|x_{0}\right|<\delta_{0}$,

(iii) globally asymptotically stable in probability if it is stochastically asymptotically stable and, moreover, for all $x_{0} \in \mathbb{R}^{n}$

$$
P\left\{\lim _{t \rightarrow \infty} x\left(t ; t_{0}, x_{0}\right)=0\right\}=1 .
$$

Lemma 2. If there exists a nonnegative function $V(x, t) \in$ $C^{2,1}\left(\mathbb{R}^{n} \times\left[t_{0}, \infty\right] ; \mathbb{R}_{+}\right)$, two continuous functions $\psi_{1}, \psi_{2}$ : $\mathbb{R}_{+}^{0} \rightarrow \mathbb{R}_{+}^{0}$, and a positive constant $K$ such that, for $|x|<K$,

$$
\psi_{1}(|x|) \leq V(x, t) \leq \psi_{2}(|x|)
$$

hold.

(i) If

$$
L V \leq 0, \quad \text { for }|x| \in[0, K]
$$

then the trivial solution of system (A.1) is stochastically stable.

(ii) If there exists a continuous function $\psi_{3}: \mathbb{R}_{+}^{0} \rightarrow \mathbb{R}_{+}^{0}$ such that

$$
L V \leq-\psi_{3}(|x|)
$$

holds, then the trivial solution of system (15) is stochastically asymptotically stable.

(iii) If (ii) holds and moreover

$$
\lim _{r \rightarrow \infty} \psi_{1}(r)=+\infty
$$

then the trivial solution of system (15) is globally asymptotically stable in probability.

For the stability of the equilibrium of a nonlinear stochastic system, it can be reduced to problems concerning stability of solutions of the linear associated system. The linear form of (15) is defined as follows:

$$
d x(t)=F(t) \cdot x(t) d t+G(t) \cdot x(t) d B(t), \quad t \geq t_{0} .
$$

Lemma 3. If the trivial solution is stochastically stable for the linear system (23) with constant coefficients $(F(t)=F$, $G(t)=G$ ) and the coefficients of systems (15) and (23) satisfy the following inequality:

$$
|f(x, t)-F \cdot x|+|g(x, t)-G \cdot x|<\rho|x|
$$

in a sufficiently small neighborhood of $x=0$, with a sufficiently small constant $\rho$, then the trivial solution of system (15) is asymptotically stable in probability. 
Consider the following $n$-dimensional stochastic functional differential equation

$$
d x=f\left(t, x_{t}\right) d t+g\left(t, x_{t}\right) d B(t)
$$

with initial condition $x_{0}=\varphi \in \mathscr{H}$, where $\mathscr{H}$ is the space of $\mathscr{F}_{0}$-adapted random variables $\varphi$, with $\varphi(s) \in \mathbb{R}^{n}$ for $s \leq 0$, and

$$
\|\varphi\|=\sup _{s \leq 0}|\varphi(s)|, \quad\|\varphi\|_{1}^{2}=\sup E\left(|\varphi(s)|^{2}\right) .
$$

Definition 4. The trivial solution of system (25) is said to be

(i) mean square stable if, for each $\varepsilon>0$, there exists $\delta(\varepsilon)>0$ such that for any initial process $\varphi(\theta)$,

$$
E\left(|x(t, \varphi(\theta))|^{2}\right)<\varepsilon
$$

for any $t \geq 0$ provided that $\sup _{\theta \leq 0} E\left(|\varphi(\theta)|^{2}\right)<\delta(\varepsilon)$,

(ii) asymptotically mean square stable if it is mean square stable and

$$
\lim _{t \rightarrow \infty} E\left(|x(t, \varphi)|^{2}\right)=0
$$

(iii) stochastically stable if for any $\varepsilon_{1}>0$ and $\varepsilon_{2}>0$, there exists a $\delta>0$ such that

$$
P\left\{\sup _{t \geq 0}|x(t, \varphi)| \leq \varepsilon_{1}\right\} \geq 1-\varepsilon_{2}
$$

provided that $P\{\|\varphi\| \leq \delta\}=1$.

\section{Dynamical Behavior of the System without Delays}

We first study the stochastic stability of the equilibria $(0,0)$ of model (13). Throughout the paper, we assume that the basic hypotheses given in the Section 2 are satisfied. The linearized system of model (13) is

$$
\begin{gathered}
d u_{1}=\left[-\left(D+m b x^{*}\right) u_{1}+\left(\mu D_{1}-m a\right) u_{2}\right] d t \\
+\sigma_{1} u_{1} d B_{1}, \\
d u_{2}=\gamma m b x^{*} u_{1} d t+\sigma_{2} u_{2} d B_{2} .
\end{gathered}
$$

For convenience, let

$$
p=\frac{\gamma m b x^{*}}{2\left(m a-\mu D_{1}\right)}, \quad q=\frac{\gamma m b x^{*}-p\left(m a-\mu D_{1}\right)}{\gamma^{2}\left(m a-\mu D_{1}\right)+\gamma D} .
$$

For linearized system (30), we have the following theorem.

Theorem 5. Let condition (6) hold. If

$$
\sigma_{1}^{2}<2 D+2 m b x^{*}, \quad \sigma_{2}^{2}<\frac{2 q}{1+q} \gamma\left(m a-\mu D_{1}\right)
$$

then the trivial solution of system (30) is globally asymptotically stable in probability.
Proof. Define a smooth function $V: \mathbb{R}^{2} \rightarrow \mathbb{R}_{+}$by

$$
V\left(u_{1}, u_{2}\right)=p u_{1}^{2}+u_{2}^{2}+q\left(\gamma u_{1}+u_{2}\right)^{2} .
$$

Then using Itô's formula, for all $\left(u_{1}, u_{2}\right) \neq(0,0)$, we have

$$
\begin{aligned}
d V\left(u_{1}, u_{2}\right)= & 2 p u_{1} d u_{1}+p\left(d u_{1}\right)^{2}+2 u_{2} d u_{2}+\left(d u_{2}\right)^{2} \\
& +2 q\left(\gamma u_{1}+u_{2}\right) d\left(\gamma u_{1}+u_{2}\right) \\
& +q\left(d\left(\gamma u_{1}+u_{2}\right)\right)^{2} \\
= & L V\left(u_{1}, u_{2}\right) d t+2 p \sigma_{1} u_{1}^{2} d B_{1}+2 \sigma_{2} u_{2}^{2} d B_{2} \\
& +2 q\left(\gamma u_{1}+u_{2}\right)\left(\gamma \sigma_{1} u_{1} d B_{1}+\sigma_{2} u_{2} d B_{2}\right),
\end{aligned}
$$

where

$$
\begin{aligned}
L V\left(u_{1}, u_{2}\right)= & 2 p u_{1}\left[-\left(D+m b x^{*}\right) u_{1}+\left(\mu D_{1}-m a\right) u_{2}\right] \\
& +p \sigma_{1}^{2} u_{1}^{2}+2 \gamma m b x^{*} u_{1} u_{2}+\sigma_{2}^{2} u_{2}^{2} \\
& +2 q\left(\gamma u_{1}+u_{2}\right) \\
\times & {\left[-\gamma D u_{1}+\gamma\left(\mu D_{1}-m a\right) u_{2}\right] } \\
& +q\left(\gamma^{2} \sigma_{1}^{2} u_{1}^{2}+\sigma_{2}^{2} u_{2}^{2}\right) \\
= & -\left[2 p\left(D+m b x^{*}\right)-p \sigma_{1}^{2}\right. \\
& \left.+2 q \gamma^{2} D-q \gamma^{2} \sigma_{1}^{2}\right] u_{1}^{2} \\
& -\left[2 q \gamma\left(m a-\mu D_{1}\right)-(1+q) \sigma_{2}^{2}\right] u_{2}^{2} \\
& -2\left[p\left(m a-\mu D_{1}\right)-\gamma m b x^{*}\right. \\
& \left.+q \gamma^{2}\left(m a-\mu D_{1}\right)+q \gamma D\right] u_{1} u_{2} .
\end{aligned}
$$

By (31), we obtain

$$
\begin{aligned}
L V & \left(u_{1}, u_{2}\right) \\
= & -\left[2 p\left(D+m b x^{*}\right)-p \sigma_{1}^{2}+2 q \gamma^{2} D-q \gamma^{2} \sigma_{1}^{2}\right] u_{1}^{2} \\
& -\left[2 q \gamma\left(m a-\mu D_{1}\right)-(1+q) \sigma_{2}^{2}\right] u_{2}^{2} .
\end{aligned}
$$

We take $\psi_{i}: R_{+}^{0} \rightarrow R_{+}^{0}(i=1,2,3)$ by

$$
\begin{gathered}
\psi_{1}(|u|)=\min \{p, 1, q\}|u|^{2}, \\
\psi_{2}(|u|)=\max \{p, 1, q\}|u|^{2}, \\
\psi_{3}(|u|)=\min \left\{2 p\left(D+m b x^{*}\right)-p \sigma_{1}^{2}+2 q \gamma^{2} D\right. \\
\left.-q \gamma^{2} \sigma_{1}^{2}, 2 q \gamma\left(m a-\mu D_{1}\right)-(1+q) \sigma_{2}^{2}\right\}|u|^{2} ;
\end{gathered}
$$

thus the thesis follows by Lemma 2. This completes the proof of Theorem 5 . 
Now, we are in a position to prove the stability of the trivial solution $(0,0)$ of model (13).

Theorem 6. Let condition (6) hold. If the conditions in (32) are satisfied, then the trivial solution of model (13) is stochastically asymptotically stable.

Proof. For a sufficiently small constant $\epsilon>0,\left(u_{1}, u_{2}\right) \epsilon$ $(-\epsilon, \epsilon) \times(-\epsilon, \epsilon)$, we have

$$
\begin{gathered}
|f(t, X)-F \cdot X|+|g(t, X)-G \cdot X| \\
=\sqrt{F_{1}^{2}\left(u_{1}, u_{2}\right)+F_{2}^{2}\left(u_{1}, u_{2}\right) .}
\end{gathered}
$$

Note that $F_{1}, F_{2}$ are the terms of order $\geq 2$ in $u_{1}$ and $u_{2}$; then we have

$$
\lim _{u_{1}^{2}+u_{2}^{2} \rightarrow 0} \frac{F_{1}^{2}\left(u_{1}, u_{2}\right)+F_{2}^{2}\left(u_{1}, u_{2}\right)}{u_{1}^{2}+u_{2}^{2}}=0 .
$$

Thus for a sufficiently small constant $\rho>0$, we have

$$
F_{1}^{2}\left(u_{1}, u_{2}\right)+F_{2}^{2}\left(u_{1}, u_{2}\right)<\rho^{2}\left(u_{1}^{2}+u_{2}^{2}\right)
$$

provided $u_{1}^{2}+u_{2}^{2}<\epsilon^{2}$. Therefore,

$$
|f(t, X)-F \cdot X|+|g(t, X)-G \cdot X|<\rho|u| .
$$

Applying Lemma 3 and Theorem 5, we obtain the conclusion.

\section{Dynamical Behavior of the System with Delays}

We now study the stability in probability of the equilibria $(0,0)$ of system (11). Its corresponding linearized system is

$$
\begin{aligned}
& d u_{1}=\left[-\left(D+m b x^{*}\right) u_{1}\right. \\
& \left.\quad+\mu D_{1} \int_{0}^{\infty} f(s) u_{2}(t-s) d s-m a u_{2}\right] d t \\
& +\sigma_{1} u_{1} d B_{1}, \\
& d u_{2}=\gamma m b x^{*} \int_{0}^{\infty} g(s) u_{1}(t-s) d s d t+\sigma_{2} u_{2} d B_{2} .
\end{aligned}
$$

Define the average time lags as

$$
T_{f}=\int_{0}^{\infty} s f(s) d s, \quad T_{g}=\int_{0}^{\infty} s g(s) d s
$$

and let $q$, $p$ be defined in (31). For linearized system (42) we have the following theorem.
Theorem 7. Let condition (6) hold. If

$$
\begin{aligned}
\sigma_{1}^{2}+ & 2 \mu D_{1} \gamma m b x^{*} T_{f}+\frac{1+q}{p+q \gamma^{2}}\left(D+m b x^{*}\right) \gamma m b x^{*} T_{g} \\
& <2 D+\frac{2 p m b x^{*}}{p+q \gamma^{2}} \\
\sigma_{2}^{2}+ & \left(D+m b x^{*}+2 m a+2 \mu D_{1}\right) \gamma m b x^{*} T_{g} \\
& <\frac{2 q}{1+q} \gamma\left(m a-\mu D_{1}\right),
\end{aligned}
$$

then the trivial solution of system (42) is asymptotically mean square stable.

Proof. Consider the function $V_{1}\left(u_{1}, u_{2}\right)$ defined in (33). It follows from (42) and Itô's formula that

$$
\begin{aligned}
& d V_{1}\left(u_{1}, u_{2}\right)=2 p u_{1} d u_{1}+p\left(d u_{1}\right)^{2}+2 u_{2} d u_{2} \\
& +\left(d u_{2}\right)^{2}+2 q\left(\gamma u_{1}+u_{2}\right) d\left(\gamma u_{1}+u_{2}\right) \\
& +q\left(d\left(\gamma u_{1}+u_{2}\right)\right)^{2} \\
& =\left\{2 p u _ { 1 } \left[-\left(D+m b x^{*}\right) u_{1}\right.\right. \\
& +\mu D_{1} \int_{0}^{\infty} f(s) u_{2}(t-s) d s \\
& \left.-m a u_{2}\right] \\
& +p \sigma_{1}^{2} u_{1}^{2}+2 \gamma m b x^{*} u_{2} \int_{0}^{\infty} g(s) u_{1}(t-s) d s \\
& +\sigma_{2}^{2} u_{2}^{2}+2 q\left(\gamma u_{1}+u_{2}\right) \\
& \times\left[-\gamma\left(D+m b x^{*}\right) u_{1}\right. \\
& +\gamma \mu D_{1} \int_{0}^{\infty} f(s) u_{2}(t-s) d s-\gamma m a u_{2} \\
& \left.+\gamma m b x^{*} \int_{0}^{\infty} g(s) u_{1}(t-s) d s\right] \\
& \left.+q\left(\gamma^{2} \sigma_{1}^{2} u_{1}^{2}+\sigma_{2}^{2} u_{2}^{2}\right)\right\} d t \\
& +2 \sigma_{2} u_{2}^{2} d B_{2}+2 q\left(\gamma u_{1}+u_{2}\right) \\
& \times\left(\gamma \sigma_{1} u_{1} d B_{1}+\sigma_{2} u_{2} d B_{2}\right)+2 p \sigma_{1} u_{1}^{2} d B_{1}
\end{aligned}
$$


Straightforward computations lead to

$$
\begin{aligned}
L V_{1}\left(u_{1}, u_{2}\right)=- & {\left[2 p\left(D+m b x^{*}\right)-p \sigma_{1}^{2}\right.} \\
& \left.+2 q \gamma^{2}\left(D+m b x^{*}\right)-q \gamma^{2} \sigma_{1}^{2}\right] u_{1}^{2} \\
- & {\left[2 q \gamma m a-(1+q) \sigma_{2}^{2}\right] u_{2}^{2} } \\
- & 2\left[p m a+q \gamma^{2} m a+q \gamma\left(D+m b x^{*}\right)\right] u_{1} u_{2} \\
+ & 2(1+q) \gamma m b x^{*} u_{2} \int_{0}^{\infty} g(s) u_{1}(t-s) d s \\
& +2 q \gamma^{2} m b x^{*} u_{1} \int_{0}^{\infty} g(s) u_{1}(t-s) d s \\
& +2\left(p+q \gamma^{2}\right) \mu D_{1} u_{1} \int_{0}^{\infty} f(s) u_{2}(t-s) d s \\
& +2 q \gamma \mu D_{1} u_{2} \int_{0}^{\infty} f(s) u_{2}(t-s) d s .
\end{aligned}
$$

From the terms of the right-hand side of (46), we have

$$
\begin{aligned}
& u_{1} \int_{0}^{\infty} g(s) u_{1}(t-s) d s \leq \frac{1}{2}\left(u_{1}^{2}+\int_{0}^{\infty} g(s) u_{1}^{2}(t-s) d s\right), \\
& u_{2} \int_{0}^{\infty} f(s) u_{2}(t-s) d s \leq \frac{1}{2}\left(u_{2}^{2}+\int_{0}^{\infty} f(s) u_{2}^{2}(t-s) d s\right) .
\end{aligned}
$$

For the term $u_{1} \int_{0}^{\infty} f(s) u_{2}(t-s) d s$, it is clear that

$$
\begin{aligned}
u_{1} \int_{0}^{\infty} & f(s) u_{2}(t-s) d s \\
= & u_{1} u_{2}-u_{1} \int_{0}^{t} f(s) \int_{t-s}^{t} d u_{2}(\tau) d s+h_{1}(t) \\
= & u_{1} u_{2}-\gamma m b x^{*} H_{1}\left(u_{1}, u_{2}\right)+h_{1}(t) \\
& -u_{1} \int_{0}^{t} f(s) \int_{t-s}^{t} \sigma_{2} u_{2}(\tau) d B_{2}(\tau) d s,
\end{aligned}
$$

where

$$
h_{1}(t)=-u_{1} \int_{t}^{\infty} f(s)\left(u_{2}(t)-u_{2}(t-s)\right) d s,
$$

$$
\begin{array}{r}
H_{1}\left(u_{1}, u_{2}\right)=u_{1} \int_{0}^{t} f(s) \int_{t-s}^{t} \int_{0}^{\infty} g(v) u_{1}(\tau-v) d v d \tau d s \\
\leq \frac{1}{2} \int_{0}^{\infty} f(s) \\
\quad \times \int_{t-s}^{t} \int_{0}^{\infty} g(v) \\
\quad \times\left(u_{1}^{2}(t)+u_{1}^{2}(\tau-v)\right) d v d \tau d s
\end{array}
$$

$$
\begin{aligned}
=\frac{1}{2} T_{f} u_{1}^{2}+\frac{1}{2} \int_{0}^{\infty} f(s) & \\
& \times \int_{t-s}^{t} \int_{0}^{\infty} g(v) \\
& \times u_{1}^{2}(\tau-v) d v d \tau d s .
\end{aligned}
$$

For the term $u_{2} \int_{0}^{\infty} g(s) u_{1}(t-s) d s$, we have that

$$
\begin{aligned}
u_{2} \int_{0}^{\infty} g(s) u_{1}(t-s) d s \\
=u_{1} u_{2}-u_{2} \int_{0}^{t} g(s) \int_{t-s}^{t} d u_{1}(\tau) d s+h_{2}(t) \\
=u_{1} u_{2}+\left(D+m b x^{*}\right) H_{2}\left(u_{1}, u_{2}\right) \\
\quad+m a H_{3}\left(u_{1}, u_{2}\right)-\mu D_{1} H_{4}\left(u_{1}, u_{2}\right) \\
\quad+u_{2} \int_{0}^{\infty} g(s) \int_{t-s}^{t} \sigma_{2} u_{2}(\tau) d B_{2}(\tau) d s+h_{2}(t),
\end{aligned}
$$

where

$$
\begin{gathered}
h_{2}(t)=-u_{2} \int_{t}^{\infty} g(s)\left(u_{1}(t)-u_{1}(t-s)\right) d s \\
H_{2}\left(u_{1}, u_{2}\right)=u_{2} \int_{0}^{t} g(s) \int_{t-s}^{t} u_{1}(\tau) d \tau d s \\
\leq \frac{1}{2} T_{g} u_{2}^{2}+\frac{1}{2} \int_{0}^{\infty} g(s) \int_{t-s}^{t} u_{1}^{2}(\tau) d \tau d s \\
H_{3}\left(u_{1}, u_{2}\right)=u_{2} \int_{0}^{t} g(s) \int_{t-s}^{t} u_{2}(\tau) d \tau d s \\
\leq \frac{1}{2} T_{g} u_{2}^{2}+\frac{1}{2} \int_{0}^{\infty} g(s) \int_{t-s}^{t} u_{2}^{2}(\tau) d \tau d s \\
H_{4}\left(u_{1}, u_{2}\right)= \\
u_{2} \int_{0}^{t} g(s) \int_{t-s}^{t} \int_{0}^{\infty} f(v) u_{2}(\tau-v) d v d \tau d s \\
\leq \frac{1}{2} T_{g} u_{2}^{2}+\frac{1}{2} \int_{0}^{\infty} g(s) \\
\times \int_{t-s}^{t} \int_{0}^{\infty} f(v)
\end{gathered}
$$

Substituting (47)-(48) together with (51) into (46), we obtain

$$
\begin{aligned}
L V_{1}\left(u_{1}, u_{2}\right) \leq-[ & 2 p\left(D+m b x^{*}\right)-p \sigma_{1}^{2} \\
& +2 q \gamma^{2}\left(D+m b x^{*}\right)-q \gamma^{2} \sigma_{1}^{2}-q \gamma^{2} m b x^{*} \\
& \left.-\left(p+q \gamma^{2}\right) \mu D_{1} \gamma m b x^{*} T_{f}\right] u_{1}^{2} \\
- & {\left[2 q \gamma m a-(1+q) \sigma_{2}^{2}-(1+q)\right.}
\end{aligned}
$$




$$
\begin{gathered}
\times\left(D+m b x^{*}+m a+\mu D_{1}\right) \gamma m b x^{*} T_{g} \\
\left.-q \gamma \mu D_{1}\right] u_{2}^{2} \\
-2\left[p m a+q \gamma^{2} m a+q \gamma\left(D+m b x^{*}\right)\right. \\
\left.\quad-(1+q) \gamma m b x^{*}-\left(p+q \gamma^{2}\right) \mu D_{1}\right] u_{1} u_{2} \\
+(1+q) \gamma m b x^{*} \\
\times\left[\mu D_{1} \int_{0}^{\infty} g(s)\right. \\
\quad \times \int_{t-s}^{t} \int_{0}^{\infty} f(v) u_{2}^{2}(\tau-v) d v d \tau d s \\
+\left(D+m b x^{*}\right) \int_{0}^{\infty} g(s) \int_{t-s}^{t} u_{1}^{2}(\tau) d \tau d s \\
\left.+m a \int_{0}^{\infty} g(s) \int_{t-s}^{t} u_{2}^{2}(\tau) d \tau d s\right] \\
+q \gamma^{2} m b x^{*} \int_{0}^{\infty} g(s) u_{1}^{2}(t-s) d s \\
+q \gamma \mu D_{1} \int_{0}^{\infty} f(s) u_{2}^{2}(t-s) d s \\
+\left(p+q \gamma^{2}\right) \mu D_{1} \gamma m b x^{*} \\
\times \int_{0}^{\infty} f(s) \int_{t-s}^{t} \int_{0}^{\infty} g(v) u_{1}^{2}(\tau-v) d v d \tau d s \\
+2(1+q) \gamma m b x^{*} h_{2}(t) \\
+2\left(p+q \gamma^{2}\right) \mu D_{1} h_{1}(t) .
\end{gathered}
$$

For technical reasons, we assume that $\int_{0}^{\infty} s^{2} f(s) d s<\infty$ and $\int_{0}^{\infty} s^{2} g(s) d s<\infty$. Then the function

$$
\begin{aligned}
& V_{2}\left(u_{1}, u_{2}\right) \\
& =(1+q) \gamma m b x^{*} \\
& \quad \times\left[\mu D_{1} \int_{0}^{\infty} g(s)\right. \\
& \quad \times \int_{t-s}^{t} \int_{r}^{t} \int_{0}^{\infty} f(v) u_{2}^{2}(\tau-v) d v d \tau d r d s \\
& \quad+\left(D+m b x^{*}\right) \\
& \quad \times \int_{0}^{\infty} g(s) \int_{t-s}^{t} \int_{r}^{t} u_{1}^{2}(\tau) d \tau d r d s \\
& \left.\quad+m a \int_{0}^{\infty} g(s) \int_{t-s}^{t} \int_{r}^{t} u_{2}^{2}(\tau) d \tau d r d s\right]
\end{aligned}
$$

$$
\begin{aligned}
& +q \gamma^{2} m b x^{*} \int_{0}^{\infty} g(s) \int_{t-s}^{t} u_{1}^{2}(\tau) d \tau d s \\
& +q \gamma \mu D_{1} \int_{0}^{\infty} f(s) \int_{t-s}^{t} u_{2}^{2}(\tau) d \tau d s \\
& +\left(p+q \gamma^{2}\right) \mu D_{1} \gamma m b x^{*} \\
& \times \int_{0}^{\infty} f(s) \\
& \quad \times \int_{t-s}^{t} \int_{r}^{t} \int_{0}^{\infty} g(v) u_{1}^{2}(\tau-v) d v d \tau d r d s
\end{aligned}
$$

is well defined. Using Itô's formula, we have

$$
\begin{aligned}
L\left(V_{1}+V_{2}\right) \leq- & {\left[2 p\left(D+m b x^{*}\right)-p \sigma_{1}^{2}\right.} \\
& +2 q \gamma^{2}\left(D+m b x^{*}\right)-q \gamma^{2} \sigma_{1}^{2}-2 q \gamma^{2} m b x^{*} \\
& -\left(p+q \gamma^{2}\right) \mu D_{1} \gamma m b x^{*} T_{f} \\
& \left.-(1+q)\left(D+m b x^{*}\right) \gamma m b x^{*} T_{g}\right] u_{1}^{2} \\
- & {\left[2 q \gamma m a-(1+q) \sigma_{2}^{2}-(1+q)\right.} \\
& \times\left(D+m b x^{*}+2 m a+\mu D_{1}\right) \gamma m b x^{*} T_{g} \\
& \left.\quad-2 q \gamma \mu D_{1}\right] u_{2}^{2} \\
- & 2\left[p m a+q \gamma^{2} m a+q \gamma\left(D+m b x^{*}\right)\right. \\
& \left.\quad-(1+q) \gamma m b x^{*}-\left(p+q \gamma^{2}\right) \mu D_{1}\right] u_{1} u_{2} \\
+ & (1+q) \gamma m b x^{*} \mu D_{1} T_{g} \\
\times & \int_{0}^{\infty} f(s) u_{2}^{2}(t-s) d s \\
+ & \left(p+q \gamma^{2}\right) \mu D_{1} \gamma m b x^{*} T_{f} \\
\times & \int_{0}^{\infty} g(s) u_{1}^{2}(t-s) d s \\
+ & 2(1+q) \gamma m b x^{*} h_{2}(t) \\
+ & 2\left(p+q \gamma^{2}\right) \mu D_{1} h_{1}(t) .
\end{aligned}
$$

We now consider the function

$$
\begin{aligned}
V_{3}\left(u_{1}, u_{2}\right)= & (1+q) \gamma m b x^{*} \mu D_{1} T_{g} \\
& \times \int_{0}^{\infty} f(s) \int_{t-s}^{t} u_{2}^{2}(\tau) d \tau d s \\
& +\left(p+q \gamma^{2}\right) \mu D_{1} \gamma m b x^{*} T_{f} \\
& \times \int_{0}^{\infty} g(s) \int_{t-s}^{t} u_{1}^{2}(\tau) d \tau d s .
\end{aligned}
$$


It follows from (56) and (57) that

$$
\begin{aligned}
& L\left(V_{1}+V_{2}+V_{3}\right) \\
& \leq-\left[2 p\left(D+m b x^{*}\right)-p \sigma_{1}^{2}\right. \\
& +2 q \gamma^{2}\left(D+m b x^{*}\right)-q \gamma^{2} \sigma_{1}^{2}-2 q \gamma^{2} m b x^{*} \\
& -2\left(p+q \gamma^{2}\right) \mu D_{1} \gamma m b x^{*} T_{f} \\
& -(1+q)\left(D+m b x^{*}\right) \gamma m b x^{*} T_{g} \\
& -2\left(p+q \gamma^{2}\right) \mu D_{1} \int_{t}^{\infty} f(s) d s \\
& \left.-(1+q) \gamma m b x^{*} \int_{t}^{\infty} g(s) d s\right] u_{1}^{2} \\
& -\left[2 q \gamma m a-(1+q) \sigma_{2}^{2}-(1+q)\right. \\
& \times\left(D+m b x^{*}+2 m a+2 \mu D_{1}\right) \gamma m b x^{*} T_{g} \\
& -2 q \gamma \mu D_{1}-\left(p+q \gamma^{2}\right) \mu D_{1} \int_{t}^{\infty} f(s) d s \\
& \left.-2(1+q) \gamma m b x^{*} \int_{t}^{\infty} g(s) d s\right] u_{2}^{2} \\
& -2\left[p m a+q \gamma^{2} m a+q \gamma\left(D+m b x^{*}\right)\right. \\
& \left.-(1+q) \gamma m b x^{*}-\left(p+q \gamma^{2}\right) \mu D_{1}\right] u_{1} u_{2} \\
& +\left(p+q \gamma^{2}\right) \mu D_{1} \int_{t}^{\infty} f(s) u_{2}^{2}(t-s) d s \\
& +(1+q) \gamma m b x^{*} \int_{t}^{\infty} g(s) u_{1}^{2}(t-s) d s .
\end{aligned}
$$

Therefore, for the function

$$
V\left(u_{1}, u_{2}\right)=V_{1}\left(u_{1}, u_{2}\right)+V_{2}\left(u_{1}, u_{2}\right)+V_{3}\left(u_{1}, u_{2}\right),
$$

we have

$$
\begin{aligned}
L V \leq-[ & 2 p\left(D+m b x^{*}\right)-p \sigma_{1}^{2} \\
& +2 q \gamma^{2}\left(D+m b x^{*}\right)-q \gamma^{2} \sigma_{1}^{2}-2 q \gamma^{2} m b x^{*} \\
& -2\left(p+q \gamma^{2}\right) \mu D_{1} \gamma m b x^{*} T_{f} \\
& -(1+q)\left(D+m b x^{*}\right) \gamma m b x^{*} T_{g} \\
& -2\left(p+q \gamma^{2}\right) \mu D_{1} \int_{t}^{\infty} f(s) d s \\
& \left.-(1+q) \gamma m b x^{*} \int_{t}^{\infty} g(s) d s\right] u_{1}^{2}
\end{aligned}
$$

By (44), we choose $\varepsilon>0$ such that

$$
\begin{aligned}
& 2 p\left(D+m b x^{*}\right)+2 q \gamma^{2} D \\
& >\quad\left(p+q \gamma^{2}\right) \sigma_{1}^{2}+2\left(p+q \gamma^{2}\right) \mu D_{1} \gamma m b x^{*} T_{f} \\
& \quad+(1+q)\left(D+m b x^{*}\right) \gamma m b x^{*} T_{g} \\
& \quad+2\left(p+q \gamma^{2}\right) \mu D_{1} \varepsilon+(1+q) \gamma m b x^{*} \varepsilon \\
& \frac{2 q}{1+q} \gamma\left(m a-\mu D_{1}\right) \\
& >\sigma_{2}^{2}+\left(D+m b x^{*}+2 m a+2 \mu D_{1}\right) \gamma m b x^{*} T_{g} \\
& \quad+\left(p+q \gamma^{2}\right) \mu D_{1} \varepsilon+2(1+q) \gamma m b x^{*} \epsilon .
\end{aligned}
$$

Let $T=T(\varepsilon)$ such that $\int_{t}^{\infty} f(s) d s<\varepsilon$ and $\int_{t}^{\infty} g(s) d s<\varepsilon$ for all $t \geq T$. Then for all $t \geq T$, one has

$$
\begin{aligned}
L V \leq-[ & 2 p\left(D+m b x^{*}\right)-p \sigma_{1}^{2}+2 q \gamma^{2}\left(D+m b x^{*}\right) \\
& -q \gamma^{2} \sigma_{1}^{2}-2 q \gamma^{2} m b x^{*} \\
& -2\left(p+q \gamma^{2}\right) \mu D_{1} \gamma m b x^{*} T_{f}-(1+q) \\
& \times\left(D+m b x^{*}\right) \gamma m b x^{*} T_{g} \\
& \left.-2\left(p+q \gamma^{2}\right) \mu D_{1} \varepsilon-(1+q) \gamma m b x^{*} \varepsilon\right] u_{1}^{2} \\
-[ & 2 q \gamma m a-(1+q) \sigma_{2}^{2}-(1+q) \\
& \times\left(D+m b x^{*}+2 m a+2 \mu D_{1}\right) \gamma m b x^{*} T_{g} \\
& -2 q \gamma \mu D_{1}-\left(p+q \gamma^{2}\right) \mu D_{1} \varepsilon \\
& \left.-2(1+q) \gamma m b x^{*} \varepsilon\right] u_{2}^{2} \\
+ & \left(p+q \gamma^{2}\right) \mu D_{1}\left\|\varphi_{2}\right\|^{2} \int_{t}^{\infty} f(s) d s \\
+ & (1+q) \gamma m b x^{*}\left\|\varphi_{1}\right\|^{2} \int_{t}^{\infty} g(s) d s .
\end{aligned}
$$


For convenience, let

$$
\begin{array}{rl}
Q=\min \{ & 2 p\left(D+m b x^{*}\right)-p \sigma_{1}^{2} \\
& +2 q \gamma^{2}\left(D+m b x^{*}\right)-q \gamma^{2} \sigma_{1}^{2}-2 q \gamma^{2} m b x^{*} \\
& -2\left(p+q \gamma^{2}\right) \mu D_{1} \gamma m b x^{*} T_{f}-(1+q) \\
& \times\left(D+m b x^{*}\right) \gamma m b x^{*} T_{g} \\
& -2\left(p+q \gamma^{2}\right) \mu D_{1} \varepsilon-(1+q) \gamma m b x^{*} \varepsilon \\
2 & q \gamma m a-(1+q) \sigma_{2}^{2}-(1+q) \\
& \times\left(D+m b x^{*}+2 m a+2 \mu D_{1}\right) \gamma m b x^{*} T_{g} \\
& -2 q \gamma \mu D_{1}-\left(p+q \gamma^{2}\right) \mu D_{1} \varepsilon \\
& \left.-2(1+q) \gamma m b x^{*} \varepsilon\right\} .
\end{array}
$$

Integrating both sides of (62) from $T$ to $t \geq T$, we have

$$
\begin{aligned}
E(V(t))+Q \int_{T}^{t} E\left(u_{1}^{2}(s)+u_{2}^{2}(s)\right) d s \\
\leq V(T)+\left(p+q \gamma^{2}\right) \mu D_{1}\left\|\varphi_{2}\right\|^{2} \int_{T}^{t} \int_{s}^{\infty} f(u) d u d s \\
\quad+(1+q) \gamma m b x^{*}\left\|\varphi_{1}\right\|^{2} \int_{T}^{t} \int_{t}^{\infty} g(u) d u d s \\
\leq V(T)+\left(p+q \gamma^{2}\right) \mu D_{1}\left\|\varphi_{2}\right\|^{2} \int_{0}^{\infty} s f(s) d s \\
\quad+(1+q) \gamma m b x^{*}\left\|\varphi_{1}\right\|^{2} \int_{0}^{\infty} s g(s) d s \\
=V(T)+\left(p+q \gamma^{2}\right) \mu D_{1}\left\|\varphi_{2}\right\|^{2} T_{f} \\
\quad+(1+q) \gamma m b x^{*}\left\|\varphi_{1}\right\|^{2} T_{g}<\infty .
\end{aligned}
$$

Discussing as that in He et al. [18], by the Barbălat lemma, we conclude $E\left(u_{1}^{2}(t)+u_{2}^{2}(t)\right) \rightarrow 0$ as $t \rightarrow \infty$. Applying Definition 4, we obtain the conclusion.

Now, we are in a position to prove the stability of the trivial solution $(0,0)$ of nonlinear system (11) using the Lyapunov functionals constructed above.

Theorem 8. Let condition (6) hold. If conditions (44) are satisfied, then the trivial solution $(0,0)$ of the system (11) or the equilibrium $\left(S^{*}, x^{*}\right)$ of system (6) is stochastically stable.

Proof. Consider the Lyapunov function $V_{1}\left(u_{1}, u_{2}\right)$ defined in (33). It follows from (11) and Itô's formula that

$$
\begin{aligned}
d V_{1}\left(u_{1}, u_{2}\right)= & 2 p u_{1} d u_{1}+p\left(d u_{1}\right)^{2}+2 u_{2} d u_{2} \\
& +\left(d u_{2}\right)^{2}+2 q\left(\gamma u_{1}+u_{2}\right) d\left(\gamma u_{1}+u_{2}\right)
\end{aligned}
$$

$$
\begin{aligned}
& +q\left(d\left(\gamma u_{1}+u_{2}\right)\right)^{2} \\
& =\left\{2 p u _ { 1 } \left[-\left(D+m b x^{*}\right) u_{1}\right.\right. \\
& +\mu D_{1} \int_{0}^{\infty} f(s) u_{2}(t-s) d s \\
& \left.-\mathrm{mau}_{2}+F_{1}\right]+p \sigma_{1}^{2} u_{1}^{2} \\
& +2 \gamma m b x^{*} u_{2} \int_{0}^{\infty} g(s) u_{1}(t-s) d s \\
& +2 u_{2} \widetilde{F}_{2}+\sigma_{2}^{2} u_{2}^{2}+2 q\left(\gamma u_{1}+u_{2}\right) \\
& \times\left[-\gamma\left(D+m b x^{*}\right) u_{1}\right. \\
& +\gamma \mu D_{1} \int_{0}^{\infty} f(s) u_{2}(t-s) d s \\
& -\gamma m a u_{2}+\gamma F_{1} \\
& \left.+\gamma m b x^{*} \int_{0}^{\infty} g(s) u_{1}(t-s) d s+\widetilde{F}_{2}\right] \\
& \left.+q\left(\gamma^{2} \sigma_{1}^{2} u_{1}^{2}+\sigma_{2}^{2} u_{2}^{2}\right)\right\} d t \\
& +2 \sigma_{2} u_{2}^{2} d B_{2} \\
& +2 q\left(\gamma u_{1}+u_{2}\right)\left(\gamma \sigma_{1} u_{1} d B_{1}+\sigma_{2} u_{2} d B_{2}\right) \\
& +2 p \sigma_{1} u_{1}^{2} d B_{1}=L V_{1}\left(u_{1}, u_{2}\right) d t+2 \sigma_{2} u_{2}^{2} d B_{2} \\
& +2 q\left(\gamma u_{1}+u_{2}\right)\left(\gamma \sigma_{1} u_{1} d B_{1}+\sigma_{2} u_{2} d B_{2}\right) \\
& +2 p \sigma_{1} u_{1}^{2} d B_{1}
\end{aligned}
$$

where

$$
\begin{aligned}
L V_{1}\left(u_{1}, u_{2}\right)=- & {\left[2 p\left(D+m b x^{*}\right)-p \sigma_{1}^{2}\right.} \\
& \left.+2 q \gamma^{2}\left(D+m b x^{*}\right)-q \gamma^{2} \sigma_{1}^{2}\right] u_{1}^{2} \\
- & {\left[2 q \gamma m a-(1+q) \sigma_{2}^{2}\right] u_{2}^{2} } \\
- & 2\left[p m a+q \gamma^{2} m a+q \gamma\left(D+m b x^{*}\right)\right] u_{1} u_{2} \\
+ & 2(1+q) \gamma m b x^{*} u_{2} \int_{0}^{\infty} g(s) u_{1}(t-s) d s \\
& +2 q \gamma^{2} m b x^{*} u_{1} \int_{0}^{\infty} g(s) u_{1}(t-s) d s \\
& +2\left(p+q \gamma^{2}\right) \mu D_{1} u_{1} \int_{0}^{\infty} f(s) u_{2}(t-s) d s \\
& +2 q \gamma \mu D_{1} u_{2} \int_{0}^{\infty} f(s) u_{2}(t-s) d s
\end{aligned}
$$




$$
\begin{aligned}
& +2 p u_{1} F_{1}+2 u_{2} \widetilde{F}_{2} \\
& +2 q\left(\gamma u_{1}+u_{2}\right)\left(\gamma F_{1}+\widetilde{F}_{2}\right) .
\end{aligned}
$$

From the terms of the right-hand side of (66), we observe that

$$
\begin{aligned}
u_{1} \int_{0}^{\infty} f(s) u_{2}(t-s) d s \\
=u_{1} u_{2}-u_{1} \int_{0}^{t} f(s) \int_{t-s}^{t} d u_{2}(\tau) d s+h_{1}(t) \\
=u_{1} u_{2}-\gamma m b x^{*} H_{1}\left(u_{1}, u_{2}\right)-u_{1} \int_{0}^{t} f(s) \int_{t-s}^{t} \widetilde{F}_{2} d \tau d s \\
\quad-u_{1} \int_{0}^{t} f(s) \int_{t-s}^{t} \sigma_{2} u_{2}(\tau) d B_{2}(\tau) d s+h_{1}(t),
\end{aligned}
$$

where $h_{1}(t)$ is defined in (49), and

$$
\begin{aligned}
u_{2} \int_{0}^{\infty} g(s) u_{1}(t-s) d s \\
=u_{1} u_{2}-u_{2} \int_{0}^{t} g(s) \int_{t-s}^{t} d u_{1}(\tau) d s+h_{2}(t) \\
=u_{1} u_{2}+\left(D+m b x^{*}\right) H_{2}\left(u_{1}, u_{2}\right) \\
\quad+m a H_{3}\left(u_{1}, u_{2}\right)-\mu D_{1} H_{4}\left(u_{1}, u_{2}\right) \\
\quad-u_{2} \int_{0}^{t} g(s) \int_{t-s}^{t} F_{1} d \tau d s \\
\quad+u_{2} \int_{0}^{t} g(s) \int_{t-s}^{t} \sigma_{2} u_{2}(\tau) d B_{2}(\tau) d s+h_{2}(t)
\end{aligned}
$$

where $h_{2}(t)$ is defined in (52). Substituting (67) and (68) into (46), we get

$$
\begin{aligned}
L V_{1}\left(u_{1}, u_{2}\right) & \\
\leq-[ & 2 p\left(D+m b x^{*}\right)-p \sigma_{1}^{2} \\
& +2 q \gamma^{2}\left(D+m b x^{*}\right)-q \gamma^{2} \sigma_{1}^{2}-q \gamma^{2} m b x^{*} \\
& \left.-\left(p+q \gamma^{2}\right) \mu D_{1} \gamma m b x^{*} T_{f}\right] u_{1}^{2} \\
-[ & 2 q \gamma m a-(1+q) \sigma_{2}^{2}-(1+q) \\
& \times\left(D+m b x^{*}+m a+\mu D_{1}\right) \gamma m b x^{*} T_{g} \\
& \left.-q \gamma \mu D_{1}\right] u_{2}^{2} \\
-2[ & p m a+q \gamma^{2} m a \\
& +q \gamma\left(D+m b x^{*}\right)-(1+q) \gamma m b x^{*} \\
& \left.-\left(p+q \gamma^{2}\right) \mu D_{1}\right] u_{1} u_{2}+(1+q) \gamma m b x^{*}
\end{aligned}
$$

$$
\begin{aligned}
& \times\left[\mu D_{1} \int_{0}^{\infty} g(s) \int_{t-s}^{t} \int_{0}^{\infty} f(v) u_{2}^{2}(\tau-v) d v d \tau d s\right. \\
& +\left(D+m b x^{*}\right) \int_{0}^{\infty} g(s) \int_{t-s}^{t} u_{1}^{2}(\tau) d \tau d s \\
& \left.+m a \int_{0}^{\infty} g(s) \int_{t-s}^{t} u_{2}^{2}(\tau) d \tau d s\right] \\
& +q \gamma^{2} m b x^{*} \int_{0}^{\infty} g(s) u_{1}^{2}(t-s) d s \\
& +q \gamma \mu D_{1} \int_{0}^{\infty} f(s) u_{2}^{2}(t-s) d s \\
& +\left(p+q \gamma^{2}\right) \mu D_{1} \gamma m b x^{*} \\
& \times \int_{0}^{\infty} f(s) \int_{t-s}^{t} \int_{0}^{\infty} g(v) u_{1}^{2}(\tau-v) d v d \tau d s \\
& +2 p u_{1} F_{1}+2 u_{2} \widetilde{F}_{2}+2 q\left(\gamma u_{1}+u_{2}\right)\left(\gamma F_{1}+\widetilde{F}_{2}\right) \\
& -2\left(p+q \gamma^{2}\right) \mu D_{1} u_{1} \int_{0}^{\infty} f(s) \int_{t-s}^{t} \widetilde{F}_{2} d \tau d s \\
& +2\left(p+q \gamma^{2}\right) \mu D_{1} h_{1}(t) \\
& -2(1+q) \gamma m b x^{*} u_{2} \int_{0}^{\infty} g(s) \int_{t-s}^{t} F_{1} d \tau d s \\
& +2(1+q) \gamma m b x^{*} h_{2}(t) \text {. }
\end{aligned}
$$

For the functions $V_{2}\left(u_{1}, u_{2}\right)$ and $V_{3}\left(u_{1}, u_{2}\right)$ defined in (55) and (57), one has

$$
\begin{array}{rl}
L\left(V_{1}+\right. & \left.V_{2}+V_{3}\right) \\
\leq- & 2 p\left(D+m b x^{*}\right)-p \sigma_{1}^{2}+2 q \gamma^{2}\left(D+m b x^{*}\right) \\
& -q \gamma^{2} \sigma_{1}^{2}-2 q \gamma^{2} m b x^{*} \\
& -2\left(p+q \gamma^{2}\right) \mu D_{1} \gamma m b x^{*} T_{f}-(1+q) \\
& \left.\times\left(D+m b x^{*}\right) \gamma m b x^{*} T_{g}\right] u_{1}^{2} \\
-[ & 2 q \gamma m a-(1+q) \sigma_{2}^{2}-(1+q) \\
& \times\left(D+m b x^{*}+2 m a+2 \mu D_{1}\right) \gamma m b x^{*} T_{g} \\
& \left.-2 q \gamma \mu D_{1}\right] u_{2}^{2} \\
-2 & {\left[p m a+q \gamma^{2} m a+q \gamma\left(D+m b x^{*}\right)\right.} \\
& \left.-(1+q) \gamma m b x^{*}-\left(p+q \gamma^{2}\right) \mu D_{1}\right] u_{1} u_{2} \\
+ & 2 p u_{1} F_{1}+2 u_{2} \widetilde{F}_{2}+2 q\left(\gamma u_{1}+u_{2}\right)\left(\gamma F_{1}+\widetilde{F}_{2}\right) \\
+2 & 2\left(p+q \gamma^{2}\right) \mu D_{1} u_{1} \int_{0}^{\infty} f(s) \int_{t-s}^{t} \widetilde{F}_{2} d \tau d s \\
& 2\left(p \gamma^{2}\right) \mu D_{1} h_{1}(t)
\end{array}
$$




$$
\begin{aligned}
& -2(1+q) \gamma m b x^{*} u_{2} \int_{0}^{\infty} g(s) \int_{t-s}^{t} F_{1} d \tau d s \\
& +2(1+q) \gamma m b x^{*} h_{2}(t) .
\end{aligned}
$$

It follows from the expression of $h_{1}(t)$ and $h_{2}(t)$ that

$$
\begin{aligned}
& h_{1}(t) \leq 2 u_{1}^{2} \int_{t}^{\infty} f(s) d s+\left(u_{2}^{2}+\left\|\varphi_{2}\right\|^{2}\right) \int_{t}^{\infty} f(s) d s, \\
& h_{2}(t) \leq 2 u_{2}^{2} \int_{t}^{\infty} g(s) d s+\left(u_{1}^{2}+\left\|\varphi_{1}\right\|^{2}\right) \int_{t}^{\infty} g(s) d s .
\end{aligned}
$$

For $V\left(u_{1}, u_{2}\right)=V_{1}\left(u_{1}, u_{2}\right)+V_{2}\left(u_{1}, u_{2}\right)+V_{3}\left(u_{1}, u_{2}\right)$, one has

$$
\begin{aligned}
L V\left(u_{1}, u_{2}\right) & \\
\leq- & 2 p\left(D+m b x^{*}\right)-p \sigma_{1}^{2} \\
& +2 q \gamma^{2}\left(D+m b x^{*}\right)-q \gamma^{2} \sigma_{1}^{2}-2 q \gamma^{2} m b x^{*} \\
& -2\left(p+q \gamma^{2}\right) \mu D_{1} \gamma m b x^{*} T_{f}-(1+q) \\
& \times\left(D+m b x^{*}\right) \gamma m b x^{*} T_{g} \\
& -2\left(p+q \gamma^{2}\right) \mu D_{1} \int_{t}^{\infty} f(s) d s \\
& \left.-(1+q) \gamma m b x^{*} \int_{t}^{\infty} g(s) d s\right] u_{1}^{2} \\
- & 2 q \gamma m a-(1+q) \sigma_{2}^{2}-(1+q) \\
& \times\left(D+m b x^{*}+2 m a+2 \mu D_{1}\right) \gamma m b x^{*} T_{g} \\
& -2 q \gamma \mu D_{1}-\left(p+q \gamma^{2}\right) \mu D_{1} \int_{t}^{\infty} f(s) d s \\
- & \left.-2(1+q) \gamma m b x^{*} \int_{t}^{\infty} g(s) d s\right] u_{2}^{2} \\
+ & (1+q) \gamma m b x^{*} u_{2} \int_{0}^{\infty} g(s) \int_{t-s}^{t} F_{1} d \tau d s \\
+ & 2 p u_{1} F_{1}+2 u_{2} \widetilde{F}_{2}+2 q\left(\gamma u_{1}+u_{2}\right)\left(\gamma F_{1}+\widetilde{F}_{2}\right) \\
+ & +q \varphi_{2} \|_{t}^{2} f(s) d s
\end{aligned}
$$

Since $F_{1}$ and $\widetilde{F}_{2}$ are terms of order $\geq 2$ in $u_{1}, u_{2}$, then we have

$$
\lim _{u_{1}, u_{2} \rightarrow 0} \frac{F_{1}\left(u_{1}, u_{2}\right)}{\sqrt{u_{1}^{2}+u_{2}^{2}}}=\lim _{u_{1}, u_{2} \rightarrow 0} \frac{\widetilde{F}_{2}\left(u_{1}, u_{2}\right)}{\sqrt{u_{1}^{2}+u_{2}^{2}}}=0 .
$$

For $\varepsilon>0$, we can find a constant $\zeta \in(0,1)$ such that

$$
F_{1}\left(u_{1}, u_{2}\right) \leq \frac{\varepsilon}{\sqrt{2}} \sqrt{u_{1}^{2}+u_{2}^{2}}, \quad \widetilde{F}_{2}\left(u_{1}, u_{2}\right) \leq \frac{\varepsilon}{\sqrt{2}} \sqrt{u_{1}^{2}+u_{2}^{2}}
$$

provided that $u_{1}^{2}+u_{2}^{2} \leq 2 \zeta^{2}$. Now consider the class of processes

$$
\Psi=\left\{\varphi \in \mathscr{H} \mid P\left\{\sup _{-\infty \leq s \leq 0}|\varphi(s)|<\zeta\right\}=1\right\} .
$$

Notice that for $u_{t} \in \Psi$,

$$
\begin{aligned}
& \left|\int_{0}^{\infty} g(s) \int_{t-s}^{t} F_{1}(\tau) d \tau d s\right| \leq \varepsilon T_{g} \zeta, \\
& \left|\int_{0}^{\infty} f(s) \int_{t-s}^{t} \widetilde{F}_{2}(\tau) d \tau d s\right| \leq \varepsilon T_{f} \zeta
\end{aligned}
$$

are valid. Substituting (74)-(76) into (72), we obtain

$$
\begin{aligned}
L V\left(u_{1}, u_{2}\right) & \\
\leq- & 2 p\left(D+m b x^{*}\right)-p \sigma_{1}^{2} \\
+ & 2 q \gamma^{2}\left(D+m b x^{*}\right)-q \gamma^{2} \sigma_{1}^{2}-2 q \gamma^{2} m b x^{*} \\
& -2\left(p+q \gamma^{2}\right) \mu D_{1} \gamma m b x^{*} T_{f} \\
& -(1+q)\left(D+m b x^{*}\right) \gamma m b x^{*} T_{g} \\
& -2\left(p+q \gamma^{2}\right) \mu D_{1} \int_{t}^{\infty} f(s) d s \\
& \left.-(1+q) \gamma m b x^{*} \int_{t}^{\infty} g(s) d s\right] u_{1}^{2} \\
-[ & 2 q \gamma m a-(1+q) \sigma_{2}^{2}-(1+q) \\
& \times\left(D+m b x^{*}+2 m a+2 \mu D_{1}\right) \gamma m b x^{*} T_{g} \\
& -2 q \gamma \mu D_{1}-\left(p+q \gamma^{2}\right) \mu D_{1} \int_{t}^{\infty} f(s) d s \\
& \left.-2(1+q) \gamma m b x^{*} \int_{t}^{\infty} g(s) d s\right] u_{2}^{2} \\
+ & +(1+q) \gamma m b x^{*}\left\|\varphi_{1}\right\|^{2} \int_{t}^{\infty} g(s) d s \\
+ & \left.+q \gamma^{2}\right) \mu D_{1}\left\|\varphi_{2}\right\|^{2} \int_{t}^{\infty} f(s) d s \\
& \left.+\left(p+q \gamma^{2}\right) \mu D_{1} T_{f}\right] \zeta^{2} . \\
+ & (\gamma+q)^{2}+(1+q) \gamma m b x^{*} T_{g} \\
+ &
\end{aligned}
$$


Integrating both sides of the above formula from $T$ to $t \wedge T_{\varepsilon_{1}}$ yields

$$
\begin{aligned}
E\left(V\left(t \wedge T_{\varepsilon_{1}}\right)\right) \leq & V(T)+\left(p+q \gamma^{2}\right) \mu D_{1}\left\|\varphi_{2}\right\|^{2} \\
& \times \int_{0}^{t \wedge T_{\varepsilon_{1}}} \int_{s}^{\infty} f(\tau) d \tau d s \\
& +(1+q) \gamma m b x^{*}\left\|\varphi_{1}\right\|^{2} \\
& \times \int_{0}^{t \wedge T_{\varepsilon_{1}}} \int_{s}^{\infty} g(\tau) d \tau d s+2 \varepsilon k_{1} \zeta^{2} \\
\leq & V(T)+\left(p+q \gamma^{2}\right) \mu D_{1}\left\|\varphi_{2}\right\|^{2} \int_{0}^{\infty} s f(s) d s \\
& +(1+q) \gamma m b x^{*}\left\|\varphi_{1}\right\|^{2} \\
& \times \int_{0}^{\infty} s g(s) d s+2 \varepsilon k_{1} \zeta^{2},
\end{aligned}
$$

where

$$
\begin{aligned}
k_{1}= & p+1+q(\gamma+1)^{2}+(1+q) \gamma m b x^{*} T_{g} \\
& +\left(p+q \gamma^{2}\right) \mu D_{1} T_{f} .
\end{aligned}
$$

By the definition of function $V\left(u_{1}, u_{2}\right)$, we can find a constant $k_{2}>0$ such that

$$
V(T) \leq k_{2}\left(\left\|\varphi_{1}\right\|^{2}+\left\|\varphi_{2}\right\|^{2}\right) .
$$

Obviously,

$$
E\left(V\left(t \wedge T_{\varepsilon_{1}}\right)\right) \leq k_{3}\left(\left\|\varphi_{1}\right\|^{2}+\left\|\varphi_{2}\right\|^{2}\right)+2 \varepsilon k_{1} \zeta^{2},
$$

where $k_{3}=\max \left\{k_{2}+(1+q) \gamma m b x^{*}, k_{2}+\left(p+q \gamma^{2}\right) \mu D_{1}\right\}$. Now for $\varepsilon_{1}, \varepsilon_{2} \in(0,1)$, let

$$
\delta=\min \left\{\left(\frac{1 \wedge p}{2 \varepsilon k_{1}+k_{3}} \varepsilon_{2}\right)^{1 / 2} \varepsilon_{1}, \frac{\varepsilon_{1}}{2}, \frac{\zeta}{2}\right\}
$$

and $\left\|\varphi_{1}\right\|^{2}+\left\|\varphi_{2}\right\|^{2}<\delta^{2}$. Then it follows that

$$
E\left(V\left(t \wedge T_{\varepsilon_{1}}\right)\right) \leq\left(2 \varepsilon k_{1}+k_{3}\right) \delta^{2} \leq(1 \wedge p) \varepsilon_{1}^{2} \varepsilon_{2} .
$$

On the other hand, we have

$$
\begin{aligned}
E\left(V\left(t \wedge T_{\varepsilon_{1}}\right)\right) & \geq E\left[1_{\left\{T_{\varepsilon_{1}} \leq t\right\}} V\left(t \wedge T_{\varepsilon_{1}}\right)\right] \\
& =E\left[1_{\left\{T_{\varepsilon_{1}} \leq t\right\}} V\left(T_{\varepsilon_{1}}\right)\right] \\
& =P\left\{T_{\varepsilon_{1}} \leq t\right\} V\left(T_{\varepsilon_{1}}\right) \\
& \geq(1 \wedge p) \varepsilon_{1}^{2} P\left\{T_{\varepsilon_{1}} \leq t\right\} .
\end{aligned}
$$

Hence, we have $P\left\{T_{\varepsilon_{1}} \leq t\right\} \leq \varepsilon_{2}$. Let $t \rightarrow \infty$; then

$$
P\left\{T_{\varepsilon_{1}}<\infty\right\} \leq \varepsilon_{2} .
$$

Equivalently,

$$
P\left\{u_{1}^{2}+u_{2}^{2}<\varepsilon_{1}^{2}\right\} \geq 1-\varepsilon_{2} .
$$

Applying Definition 4, we obtain the conclusion.

\section{Simulations and Discussions}

In this paper, we have considered a stochastic chemostat model simulating the process of wastewater treatment. The model incorporates a general nutrient uptake function and two distributed delays. The first delay models the fact that nutrient is partially recycled after the death of the biomass by bacterial decomposition and the second indicates that the growth of the species depends on the past concentration of the nutrient. Furthermore, we consider the stochastic perturbations which are of white noise type and are proportional to the distances of $S(t), x(t)$ from the values of the positive equilibrium $S^{*}, x^{*}$. By constructing appropriate Liapunovlike functionals, some sufficient conditions for the stochastic stability of the positive equilibrium have been obtained.

For model (3), we have first analyzed the stochastic stability of the positive equilibrium $E^{*}$ in the case when the delays are ignored, that is, the average delays $T_{f}=T_{g}=0$. Our findings in Theorem 6 reveal that $E^{*}$ is stochastically stable provided that the intensities of noises are small. When at least one of the average delays $T_{f}$ and $T_{g}$ is not equal to zero, our results in Theorem 8 reveal that $E^{*}$ is stochastically stable provided that the average delays $T_{f}$ and $T_{g}$ are both small. Obviously, Theorem 8 reduces to Theorem 6 when $T_{f}=T_{g}=0$, which indicates that if the average delays are sufficiently small, $E^{*}$ is still stochastically stable; and in the case of $\sigma_{i}=0(i=1,2)$, Theorem 8 reduces to He et al. [18, Theorem 3.1]; that is to say, the equilibrium $E^{*}$ of model (3) is still stable if $\sigma_{1}$ and $\sigma_{2}$ are sufficient small, which preserves the dynamics of its corresponding deterministic counterpart (5).

To illustrate the results obtained above, some numerical simulations are carried out by using Milstein scheme [50]. Here we assume that the specific growth function $U(S)$ is of Michaelis-Menten type

$$
U(S)=\frac{S}{a_{1}+S}
$$

where $a_{1}$ is the half-saturation constant. For the kernel functions $f(s)$ and $g(s)$, we consider two special cases: (1) $f(s)=g(s)=\delta(0)$; (2) $f(s)=\alpha e^{-\alpha s}$ and $g(s)=$ $\beta e^{-\beta s}$. For case (1), the discretization of model (3) for $t=$ $0, \Delta t, 2 \Delta t, \ldots, n \Delta t$ takes the form

$$
\begin{aligned}
S_{i+1}= & S_{i}+\left[D\left(S^{0}-S_{i}\right)-m U\left(S_{i}\right) x_{i}+\mu D_{1} x_{i}\right] \Delta t \\
& +\sigma_{1}\left(S_{i}-S^{*}\right) \sqrt{\Delta t} \xi_{i}, \\
x_{i+1}= & x_{i}+x_{i}\left[-\left(D_{w}+D_{1}\right)+\gamma m U\left(S_{i}\right)\right] \Delta t \\
& +\sigma_{2}\left(x_{i}-x^{*}\right) \sqrt{\Delta t} \xi_{i},
\end{aligned}
$$

where time increment $\Delta t>0$ and $\xi_{i}$ is $N(0,1)$-distributed independent random variables which can be generated 


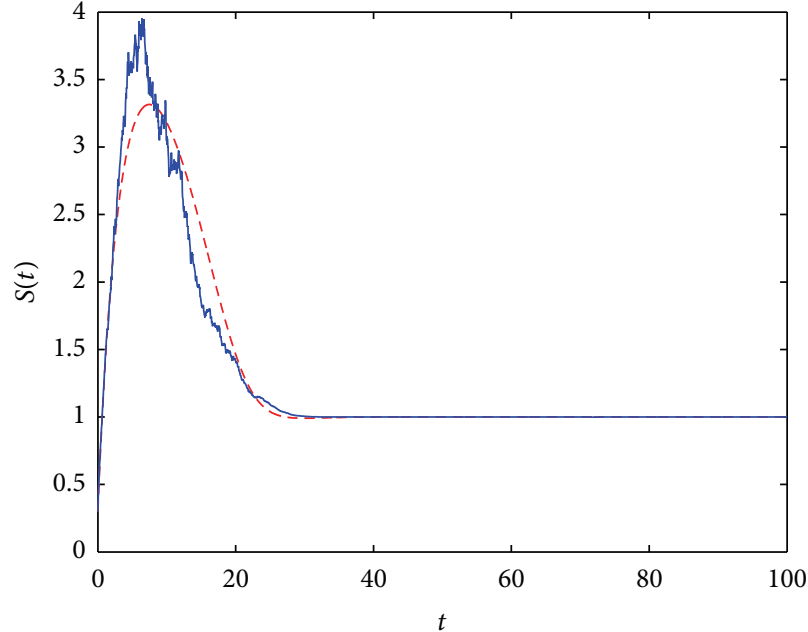

- - Deterministic trajectory

— Stochastic trajectory

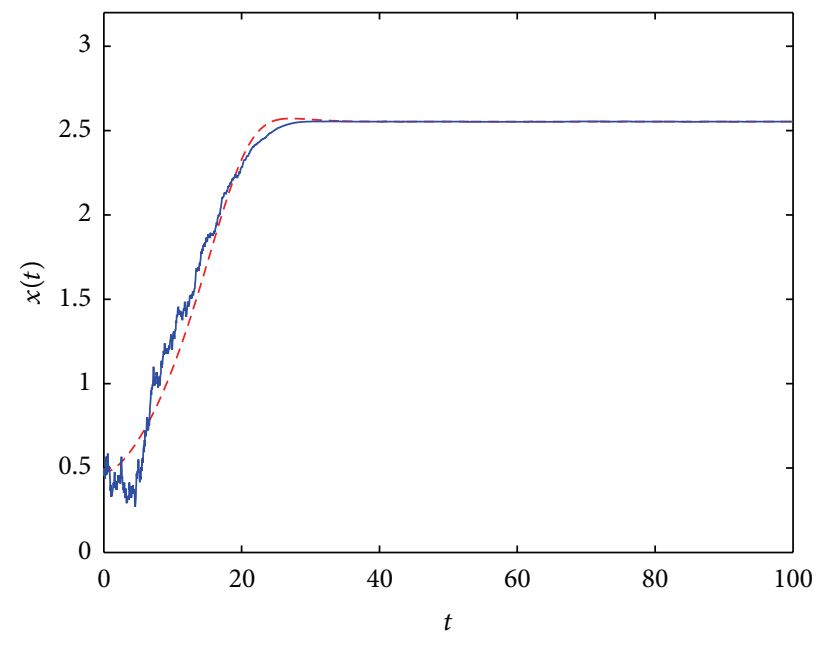

- - Deterministic trajectory

— Stochastic trajectory

FIGURE 1: The dynamics of stochastic model compared with deterministic model with $\sigma_{1}=0.1$ and $\sigma_{2}=0.08$. Here $S(0)=0.3, x(0)=0.5$.
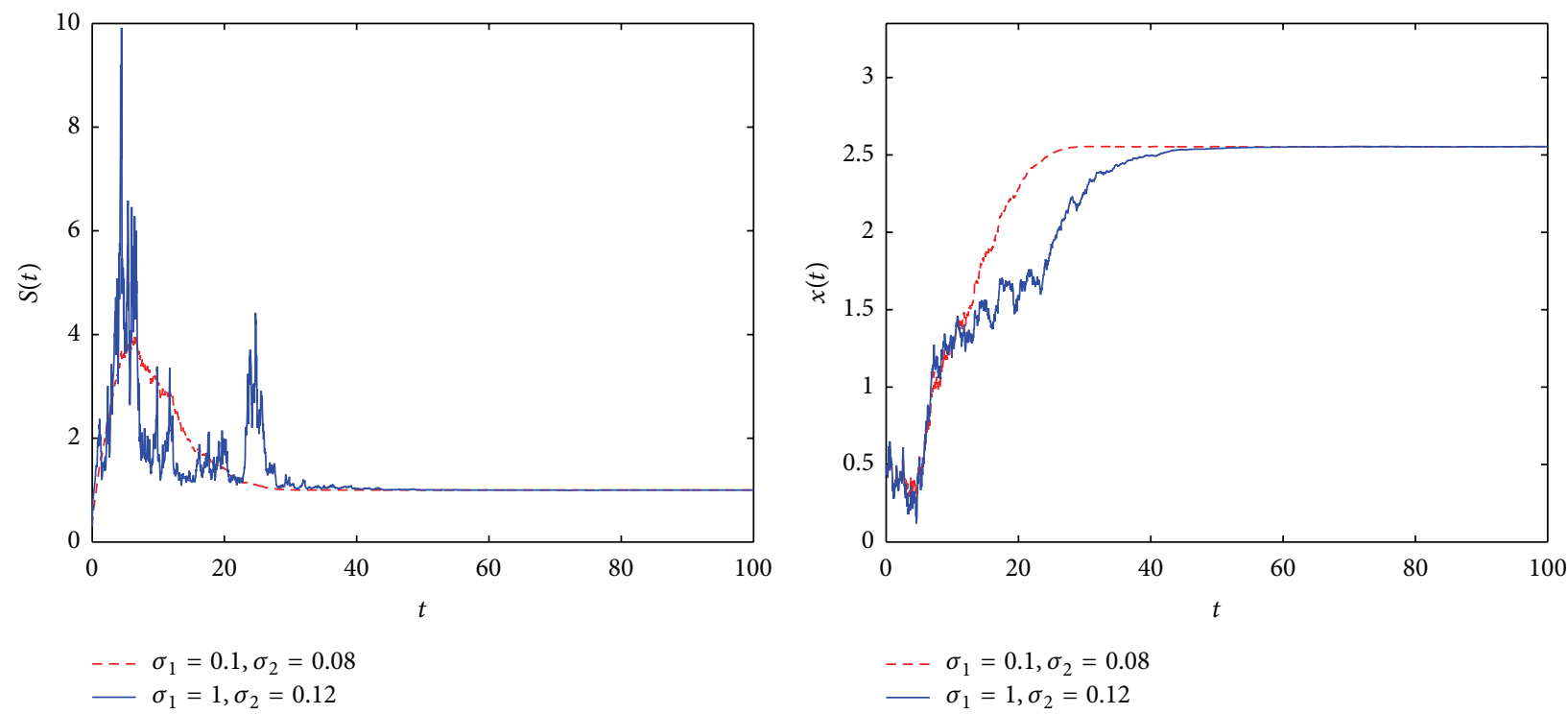

Figure 2: The dynamics of stochastic model with different values of $\sigma_{1}$ and $\sigma_{2}$. Here $S(0)=0.3, x(0)=0.5$.

numerically by pseudorandom number generators. For case (2), define

$$
\begin{gathered}
y(t)=\int_{0}^{\infty} \alpha e^{-\alpha s} x(t-s) d s, \\
z(t)=\int_{0}^{\infty} \beta e^{-\beta s} U(S(t-s)) d s,
\end{gathered}
$$

then the discretization of model (3) for $t=0, \Delta t, 2 \Delta t, \ldots, n \Delta t$ takes the form

$$
\begin{aligned}
S_{i+1}= & S_{i}+\left[D\left(S^{0}-S_{i}\right)-m U\left(S_{i}\right) x_{i}+\mu D_{1} y_{i}\right] \Delta t \\
& +\sigma_{1}\left(S_{i}-S^{*}\right) \sqrt{\Delta t} \xi_{i},
\end{aligned}
$$

$$
\begin{gathered}
x_{i+1}=x_{i}+x_{i}\left[-\left(D_{w}+D_{1}\right)+\gamma m z_{i}\right] \Delta t \\
+\sigma_{2}\left(x_{i}-x^{*}\right) \sqrt{\Delta t} \xi_{i}, \\
y_{i+1}=y_{i}+\left(-\alpha y_{i}+\alpha x_{i}\right) \Delta t, \\
z_{i+1}=z_{i}+\left(-\beta z_{i}+\beta U\left(S_{i}\right)\right) \Delta t .
\end{gathered}
$$

Let in model (3) $D=D_{w}=0.3, D_{1}=0.1, S^{0}=5, m=$ $0.7, a_{1}=0.4, \mu=0.3, \gamma=0.8$. It is easy to compute that $a \doteq 0.7143, b \doteq 0.2041, p \doteq 0.3100, q \doteq 0.2694$, and $E^{*}=$ $(1,2.55)$.

The first two examples given below concern case (1) when the delays are ignored; that is to say, it is assumed that the 

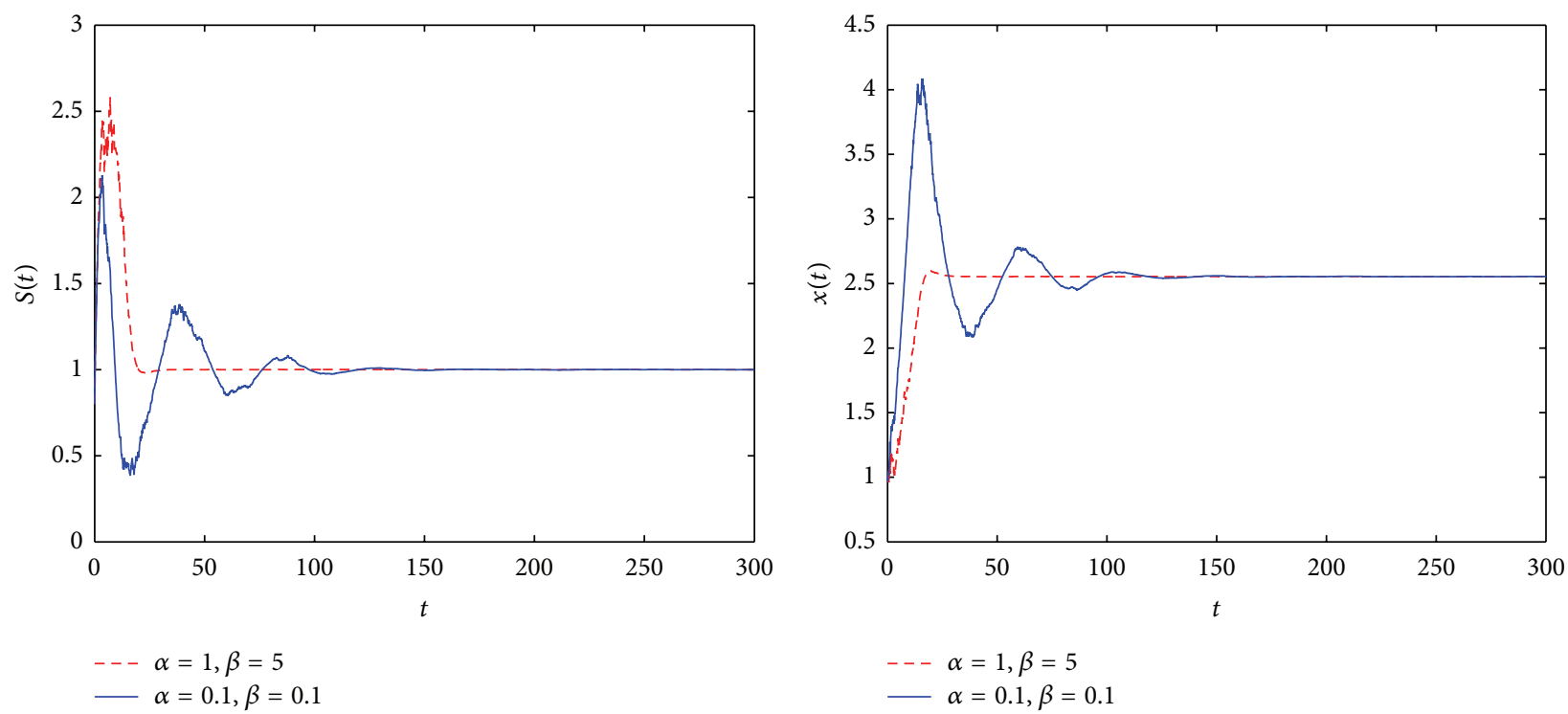

Figure 3: The dynamics of stochastic functional model with different $\alpha$, $\beta$. Here $S(0)=0.3, x(0)=0.5, y(0)=0.3, z(0)=0.5$.
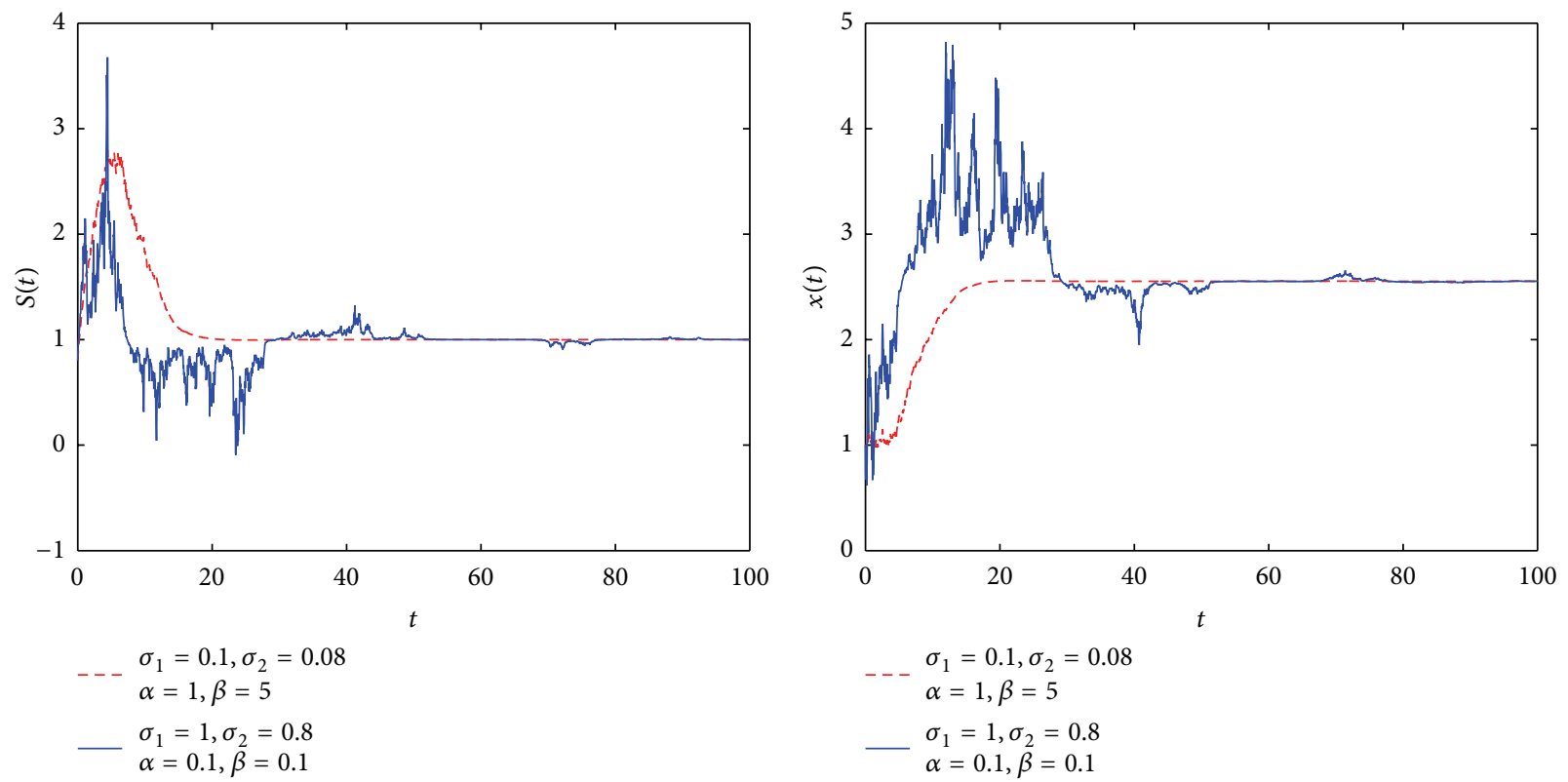

FIGURE 4: The dynamics of stochastic functional model with different $\sigma_{1}, \sigma_{2}$ and $\alpha$, $\beta$. Here $S(0)=0.3, x(0)=0.5, y(0)=0.3, z(0)=0.5$.

process of nutrient recycling and the growth response of the species are immediate and, therefore, $T_{f}=T_{g}=0$. Example 1 verifies the results obtained in Theorem 6 .

Example 1. Let $\sigma_{1}=0.1$ and $\sigma_{2}=0.08$, then by straightforward computations, we have that $0.01=\sigma_{1}^{2}<$ $2 D+2 m b x^{*} \doteq 1.3285,0.0064=\sigma_{2}^{2}<(2 q /(1+q)) \gamma(m a-$ $\left.\mu D_{1}\right) \doteq 0.1596$. In view of Theorem 6 , the equilibrium $E^{*}$ of (3) is stochastically asymptotically stable, which is consistent with the simulation results as shown in Figure 1.

To further study the combined effects of $\sigma_{i}, i=1,2$ when $T_{f}=T_{g}=0$, we need to consider four situations: (a) $\sigma_{1}$ increases, $\sigma_{2}$ increases; (b) $\sigma_{1}$ increases, $\sigma_{2}$ decreases; (c) $\sigma_{1}$ decreases, $\sigma_{2}$ increases; (d) $\sigma_{1}$ decreases, $\sigma_{2}$ decreases. Here we only give one example about situation (a); other situations can be considered similarly.

Example 2. Let the intensities $\sigma_{i}, i=1,2$ increase from $\sigma_{1}=0.1, \sigma_{2}=0.08$ to $\sigma_{1}=1, \sigma_{2}=0.12$, respectively. Simulations show that the trajectories of model (3) still approach ultimately to the positive equilibrium $E^{*}$, but they need to go through more oscillations and more time to return to $E^{*}$ (see Figure 2).

The next two examples concern case (2) when $f(s)$ and $g(s)$ take weak kernels; that is, $f(s)=\alpha e^{-\alpha s}$ and $g(s)=\beta e^{-\beta s}$, 

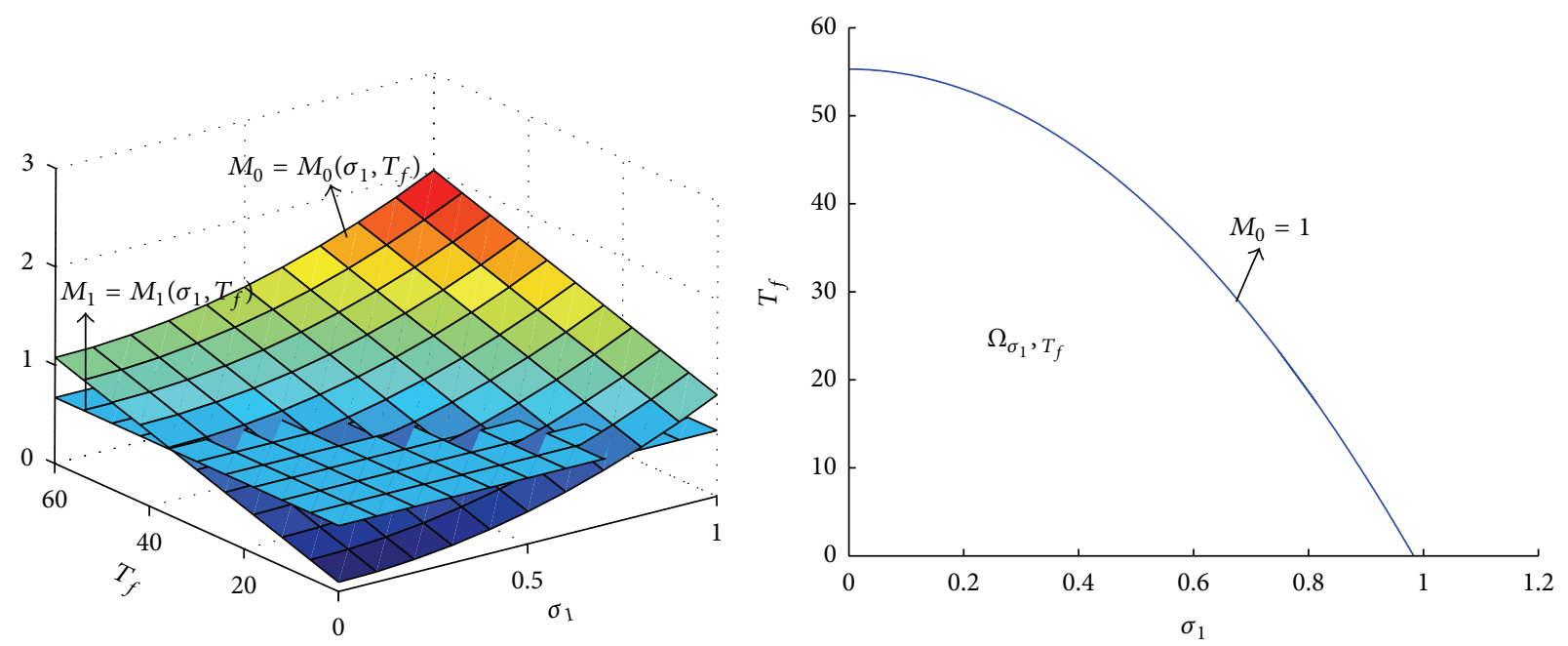

FIGURE 5: The positive equilibrium $E^{*}$ is stochastically stable provided that $\left(\sigma_{1}, T_{f}\right) \in \Omega_{\sigma_{1}, T_{f}}$. Here $\sigma_{2}=0.08$ and $T_{g}=0.2$.
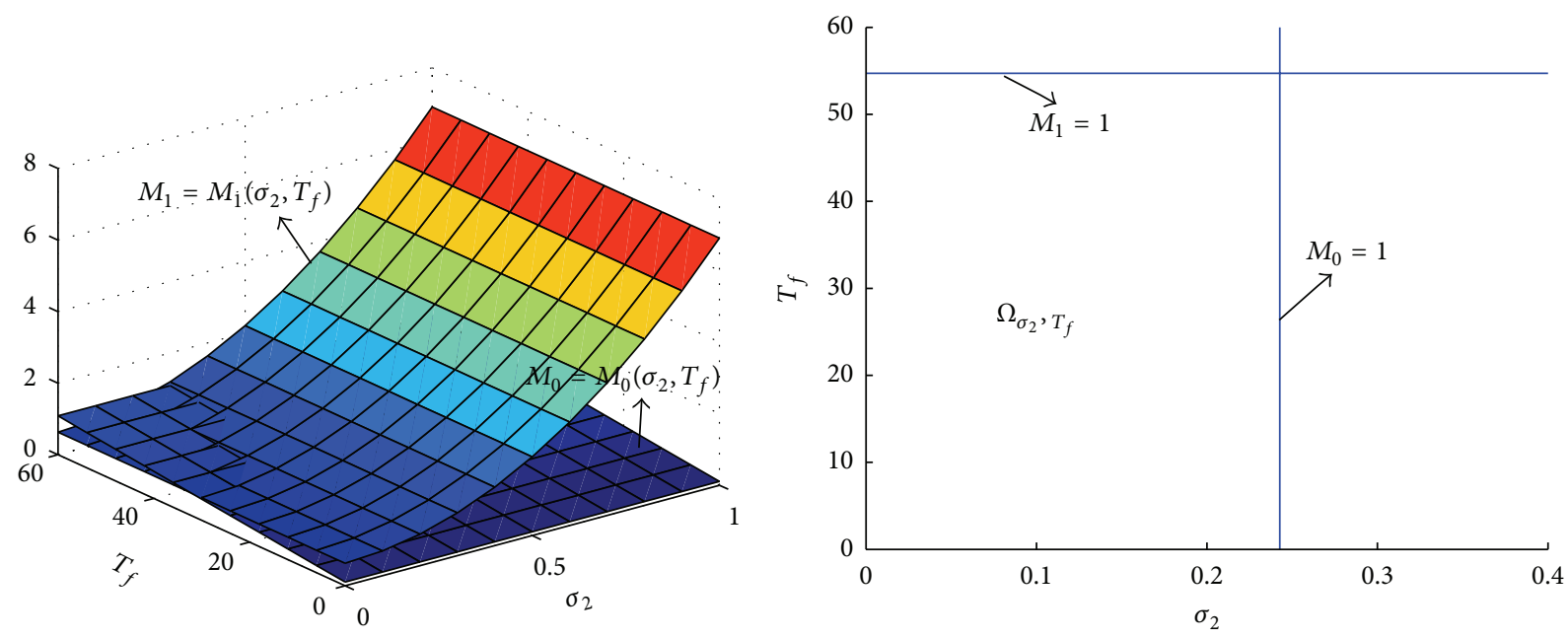

FIgURE 6: The positive equilibrium $E^{*}$ is stochastically stable provided that $\left(\sigma_{2}, T_{f}\right) \in \Omega_{\sigma_{2}, T_{f}}$. Here $\sigma_{1}=0.1$ and $T_{g}=0.2$.
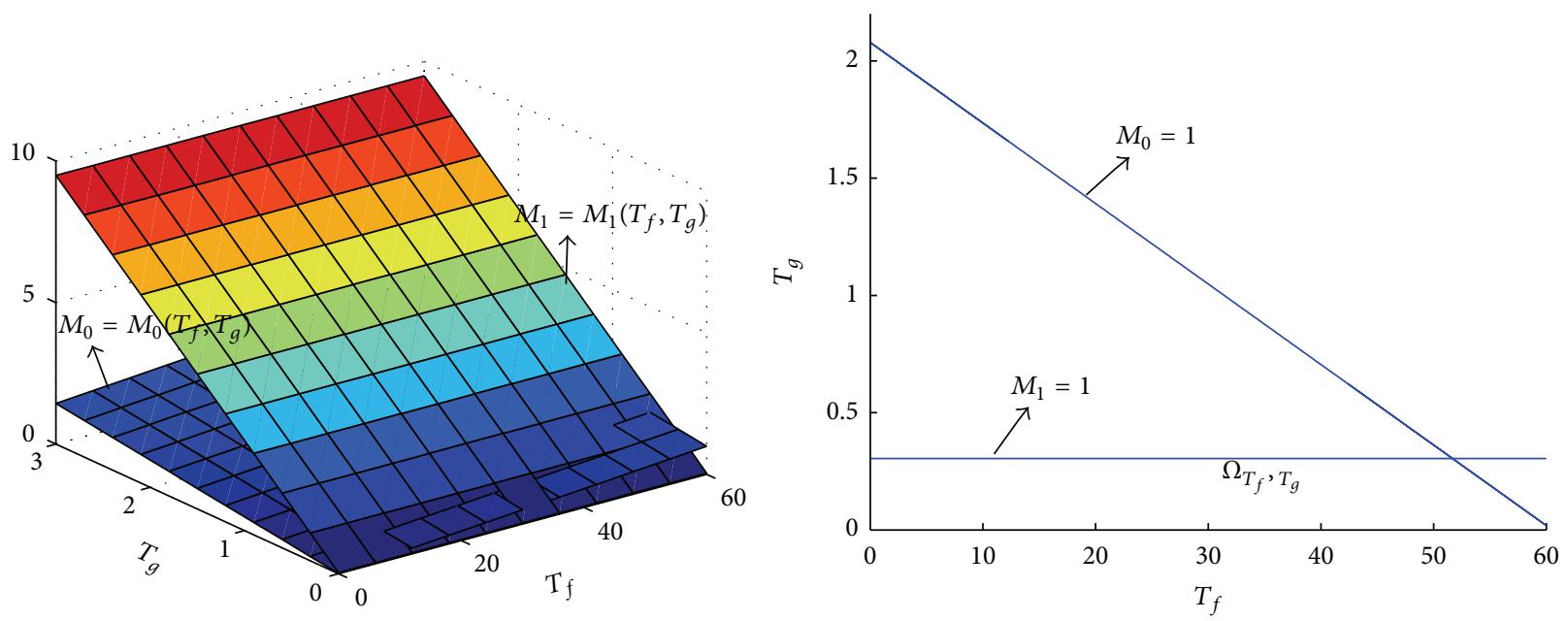

FIGURE 7: The positive equilibrium $E^{*}$ is stochastically stable provided that $\left(T_{f}, T_{g}\right) \in \Omega_{T_{f}, T_{g}}$. Here $\sigma_{1}=0.1$ and $\sigma_{2}=0.08$. 
which means that $T_{f}=1 / \alpha$ and $T_{g}=1 / \beta$. Example 3 verifies the results obtained in Theorem 8 .

Example 3. Let $\sigma_{1}=0.1, \sigma_{2}=0.08, \alpha=1$ and $\beta=5$. It is easy to compute that $\left(p+q \gamma^{2}\right) \sigma_{1}^{2}+2\left(p+q \gamma^{2}\right) \mu D_{1} \gamma m b x^{*} T_{f}+(1+$ q) $\left(D+m b x^{*}\right) \gamma m b x^{*} T_{g} \doteq 0.0624,2 p\left(D+m b x^{*}\right)+2 q \gamma^{2} D \doteq$ 0.5154 and $\sigma_{2}^{2}+\left(D+m b x^{*}+2 m a+2 \mu D_{1}\right) \gamma m b x^{*} T_{g} \doteq 0.1069$, $(2 q /(1+q)) \gamma\left(m a-\mu D_{1}\right) \doteq 0.1596$; thus conditions (44) are satisfied. By Theorem 8 , the equilibrium $E^{*}$ of model (3) is stochastically stable. Our simulation supports this result as shown in Figure 3.

To examine the combined effects of the noise intensities and the delays on the dynamics of model (3), we first consider the case when the values of $\sigma_{i}, i=1,2$ in Example 3 are fixed and the values of $\alpha$ and $\beta$ are reduced from 1 and 5 to 0.1 and 0.1 , respectively. That is to say, the average delays $T_{f}$ and $T_{g}$ increase from 1 and 0.2 to 10 and 10 , respectively. Simulation results show that the solution of (3) will suffer more oscillations and more time to approach the equilibrium $E^{*}$ when delays increase (see Figure 3 ). When both the values of the noise intensities and the delays vary, the dynamics of model (3) may become more complicated. Here we only consider the case when $\sigma_{i}(i=1,2), T_{f}$ and $T_{g}$ (i.e., $1 / \alpha$ and $1 / \beta)$ all increase. See the following Example.

Example 4. Let $\sigma_{i}(i=1,2), T_{f}$ and $T_{g}$ (i.e., $1 / \alpha$ and $\left.1 / \beta\right)$ increase from $0.1,0.08,1$, and 0.2 (i.e., $\alpha=1$ and $\beta=5$ ) to $1,0.8,10$, and 10 (i.e., $\alpha=0.1$ and $\beta=0.1$ ), respectively. It is found that the trajectories of model (3) fluctuate wildly and suffer more oscillations and need more time to approach the equilibrium $E^{*}$; please see Figure 4 .

Notice also that conditions (44) in Theorem 8 are only sufficient conditions to insure the stochastic stability of $E^{*}$, which are dependent on parameters $\sigma_{1}, \sigma_{2}, T_{f}$, and $T_{g}$. Define

$$
\begin{aligned}
M_{0}= & \left(\left(p+q \gamma^{2}\right) \sigma_{1}^{2}+2\left(p+q \gamma^{2}\right) \mu D_{1} \gamma m b x^{*} T_{f}\right. \\
& \left.+(1+q)\left(D+m b x^{*}\right) \gamma m b x^{*} T_{g}\right) \\
& \times\left(2 p\left(D+m b x^{*}\right)+2 q \gamma^{2} D\right)^{-1}, \\
M_{1}= & \frac{\sigma_{2}^{2}+\left(D+m b x^{*}+2 m a+2 \mu D_{1}\right) \gamma m b x^{*} T_{g}}{(2 q /(1+q)) \gamma\left(m a-\mu D_{1}\right)} .
\end{aligned}
$$

Thus, conditions (44) are equivalent to those when parameters $\sigma_{1}, \sigma_{2}, T_{f}$, and $T_{g}$ are seated in the following parameter set:

$$
\begin{gathered}
\Omega=\left\{\left(\sigma_{1}, \sigma_{2}, T_{f}, T_{g}\right) \mid \max \left\{M_{0}, M_{1}\right\}<1,\right. \\
\left.\sigma_{i} \geq 0, T_{f} \geq 0, T_{g} \geq 0\right\},
\end{gathered}
$$

from which we can further perform some approximate sensitivity analysis of the stochastic stability of $E^{*}$ with respect to these parameters. To do this, we can let two of the parameters (e.g., $\sigma_{1}$ and $\left.T_{f}\right)$ vary and the other two $\left(\sigma_{2}\right.$ and $T_{g}$ ) be fixed, which have six cases in all.
Let us first consider the case when $\sigma_{2}=0.08$ and $T_{g}=0.2$; then $M_{0}$ and $M_{1}$ are both functions of $\sigma_{1}$ and $T_{f}$. Then $\Omega$ defined in (92) is equivalent to

$$
\Omega_{\sigma_{1}, T_{f}}=\left\{\left(\sigma_{1}, T_{f}\right) \mid\left(\sigma_{1}, 0.08, T_{f}, 0.2\right) \in \Omega\right\},
$$

which is the projection of surfaces $M_{0}=M_{0}\left(\sigma_{1}, T_{f}\right)$ and $M_{1}=M_{1}\left(\sigma_{1}, T_{f}\right)$ in the first octant such that $\max \left\{M_{0}, M_{1}\right\}<$ 1 (see Figure 5). The positive equilibrium $E^{*}$ is stochastically stable provided that $\left(\sigma_{1}, T_{f}\right) \in \Omega_{\sigma_{1}, T_{f}}$.

To better observe the dependence of the stochastic stability of $E^{*}$ on all parameters, we further consider another two cases when $\sigma_{1}=0.1$ and $T_{g}=0.2$ are fixed and $\sigma_{1}=0.1$ and $\sigma_{2}=0.08$ are fixed. Accordingly, $\Omega$ defined in (92) is equivalent, respectively, to

$$
\begin{gathered}
\Omega_{\sigma_{2}, T_{f}}=\left\{\left(\sigma_{2}, T_{f}\right) \mid\left(0.1, \sigma_{2}, T_{f}, 0.2\right) \in \Omega\right\}, \\
\Omega_{T_{f}, T_{g}}=\left\{\left(T_{f}, T_{g}\right) \mid\left(0.1,0.08, T_{f}, T_{g}\right) \in \Omega\right\},
\end{gathered}
$$

which are plotted, respectively, in Figures 6 and 7 (other three cases can be considered similarly). From Figures 5-7, we find that the stochastic stability of $E^{*}$ is greatly affected by $\sigma_{1}, \sigma_{2}$, and $T_{g}$ and less affected by $T_{f}$ (which is consistent with the results observed in $[13,17])$. We would like to point out here that $E^{*}$ may also be stable when the parameters are seated outside of the set $\Omega$, since (44) are only sufficient conditions ensuring the stochastic stability of $E^{*}$.

In conclusion, this paper presents an investigation on the combined effect of the noises and delays on a bottom-microbe model. Our findings are useful for better understanding of the dynamics of microbial population in the activated sludge process. We should point out that there are still some other interesting topics about the wastewater treatment deserving further investigation, for example, membrane reactor, and so forth. We leave these for future considerations.

\section{Conflict of Interests}

The authors declare that there is no conflict of interests regarding the publication of this paper.

\section{Acknowledgments}

This work is supported by the National Natural Science Foundation of China (no. 11271260), Shanghai Leading Academic Discipline Project (no. XTKX2012), and the Innovation Program of Shanghai Municipal Education Commission (no. 13ZZ116).

\section{References}

[1] M. Henze, C. P. L. Grady Jr., and W. Gujer, "A general model for single-sludge wastewater treatment systems," Water Research, vol. 21, no. 5, pp. 505-515, 1987.

[2] J. C. Kabouris and A. P. Georgakakos, "Parameter and state estimation of the activated sludge process-I. Model development," Water Research, vol. 30, no. 12, pp. 2853-2865, 1996. 
[3] M. Zaiat, F. H. Passig, and E. Foresti, "A mathematical model and criteria for designing horizontal-flow anaerobic immobilized biomass reactors for wastewater treatment," Bioresource Technology, vol. 71, no. 3, pp. 235-243, 2000.

[4] G. Gehlert and J. Hapke, "Mathematical modeling of a continuous aerobic membrane bioreactor for the treatment of different kinds of wastewater," Desalination, vol. 146, no. 1-3, pp. 405-412, 2002.

[5] B. Beran and F. Kargi, "A dynamic mathematical model for wastewater stabilization ponds," Ecological Modelling, vol. 181, no. 1, pp. 39-57, 2005.

[6] Y. Peng, B. Wang, and S. Wang, "Multivariable optimal control of activated sludge process: I. Basic theory and effect of DO on operation cost," Acta Scientiae Circumstantiae, vol. 18, no. 1, pp. 11-19, 1998.

[7] Z. Chen and T. Zhang, "Long time behaviour of a stochastic model for continuous flow bioreactor," Journal of Mathematical Chemistry, vol. 51, no. 2, pp. 451-464, 2013.

[8] Z. Chen and T. Zhang, "Dynamics of a stochastic model for continuous flow bioreactor with Contois growth rate," Journal of Mathematical Chemistry, vol. 51, no. 3, pp. 1076-1091, 2013.

[9] S. Yuan, D. Xiao, and M. Han, "Competition between plasmidbearing and plasmid-free organisms in a chemostat with nutrient recycling and an inhibitor," Mathematical Biosciences, vol. 202, no. 1, pp. 1-28, 2006.

[10] J. Luo, S. Yuan, and W. Zhang, "Competition between two microorganisms in the chemostat with general variable yields and general growth rates," International Journal of Biomathematics, vol. 1, no. 4, pp. 463-474, 2008.

[11] H. L. Smith and P. Waltman, The Theory of the Chemostat: Dynamics of Microbial Competition, Cambridge University Press, Cambridge, UK, 1995.

[12] H. I. Freedman and Y. T. Xu, "Models of competition in the chemostat with instantaneous and delayed nutrient recycling," Journal of Mathematical Biology, vol. 31, no. 5, pp. 513-527, 1993.

[13] S. G. Ruan, "The effect of delays on stability and persistence in plankton models," Nonlinear Analysis. Theory, Methods \& Applications A, vol. 24, no. 4, pp. 575-585, 1995.

[14] S. Yuan, W. Zhang, and M. Han, "Global asymptotic behavior in chemostat-type competition models with delay," Nonlinear Analysis. Real World Applications, vol. 10, no. 3, pp. 1305-1320, 2009.

[15] S. Ruan and G. S. K. Wolkowicz, "Bifurcation analysis of a chemostat model with a distributed delay," Journal of Mathematical Analysis and Applications, vol. 204, no. 3, pp. 786-812, 1996.

[16] B. Li, G. S. K. Wolkowicz, and Y. Kuang, "Global asymptotic behavior of a chemostat model with two perfectly complementary resources and distributed delay," SIAM Journal on Applied Mathematics, vol. 60, no. 6, pp. 2058-2086, 2000.

[17] E. Beretta, G. I. Bischi, and F. Solimano, "Stability in chemostat equations with delayed nutrient recycling," Journal of Mathematical Biology, vol. 28, no. 1, pp. 99-111, 1990.

[18] X.-Z. He, S. Ruan, and H. Xia, "Global stability in chemostattype equations with distributed delays," SIAM Journal on Mathematical Analysis, vol. 29, no. 3, pp. 681-696, 1998.

[19] J. Jiao, X. Yang, L. Chen, and S. Cai, "Effect of delayed response in growth on the dynamics of a chemostat model with impulsive input," Chaos, Solitons and Fractals, vol. 42, no. 4, pp. 2280-2287, 2009.
[20] X. Meng, Q. Gao, and Z. Li, "The effects of delayed growth response on the dynamic behaviors of the Monod type chemostat model with impulsive input nutrient concentration," Nonlinear Analysis. Real World Applications, vol. 11, no. 5, pp. 44764486, 2010.

[21] Z. Zhao, L. Chen, and X. Song, "Extinction and permanence of chemostat model with pulsed input in a polluted environment," Communications in Nonlinear Science and Numerical Simulation, vol. 14, no. 4, pp. 1737-1745, 2009.

[22] Z. Li, L. Chen, and Z. Liu, "Periodic solution of a chemostat model with variable yield and impulsive state feedback control," Applied Mathematical Modelling, vol. 36, no. 3, pp. 1255-1266, 2012.

[23] Y. Tian, K. Sun, L. Chen, and A. Kasperski, "Studies on the dynamics of a continuous bioprocess with impulsive state feedback control," Chemical Engineering Journal, vol. 157, no. 23, pp. 558-567, 2010.

[24] O. Tagashira and T. Hara, "Delayed feedback control for a chemostat model," Mathematical Biosciences, vol. 201, no. 1-2, pp. 101-112, 2006.

[25] L. Imhof and S. Walcher, "Exclusion and persistence in deterministic and stochastic chemostat models," Journal of Differential Equations, vol. 217, no. 1, pp. 26-53, 2005.

[26] F. Campillo, M. Joannides, and I. Larramendy-Valverde, "Stochastic modeling of the chemostat," Ecological Modelling, vol. 222, no. 15, pp. 2676-2689, 2011.

[27] B. S. Khatri, A. Free, and R. J. Allen, "Oscillating microbial dynamics driven by small populations, limited nutrient supply and high death rates," Journal of Theoretical Biology, vol. 314, pp. 120-129, 2012.

[28] S. R. Hansen and S. P. Hubbell, "Single-nutrient microbial competition: qualitative agreement between experimental and theoretically forecast outcomes," Science, vol. 207, no. 4438, pp. 1491-1493, 1980.

[29] D. Porro, E. Martegani, B. M. Ranzi, and L. Alberghina, "Oscillation in continuous cultures of budding yeast: a segregated parameter analysis," Biotechnology and Bioengineering, vol. 32, no. 4, pp. 411-417, 1988.

[30] S. Yuan and M. Han, "Bifurcation analysis of a chemostat model with two distributed delays," Chaos, Solitons \& Fractals, vol. 20, no. 5, pp. 995-1004, 2004.

[31] S. Yuan, Y. Song, and M. Han, "Direction and stability of bifurcating periodic solutions of a chemostat model with two distributed delays," Chaos, Solitons \& Fractals, vol. 21, no. 5, pp. 1109-1123, 2004.

[32] S. Yuan, P. Li, and Y. Song, "Delay induced oscillations in a turbidostat with feedback control," Journal of Mathematical Chemistry, vol. 49, no. 8, pp. 1646-1666, 2011.

[33] S. Yuan and T. Zhang, "Dynamics of a plasmid chemostat model with periodic nutrient input and delayed nutrient recycling," Nonlinear Analysis. Real World Applications, vol. 13, no. 5, pp. 2104-2119, 2012.

[34] S. Yuan, W. Zhang, and Y. Zhao, "Bifurcation analysis of a model of plasmid-bearing, plasmid-free competition in a pulsed chemostat with an internal inhibitor," IMA Journal of Applied Mathematics, vol. 76, no. 2, pp. 277-297, 2011.

[35] S. Yuan, Y. Zhao, A. Xiao, and T. Zhang, "Bifurcation and chaos in a pulsed plankton model with instantaneous nutrient recycling," The Rocky Mountain Journal of Mathematics, vol. 42, no. 4, pp. 1387-1409, 2012. 
[36] P. L. Dold and G. v. R. Marais, "Evaluation of the general activated sludge model proposed by the IAWPRC task group," Water Science and Technology, vol. 18, no. 6, pp. 63-89, 1986.

[37] J. Caperon, "Time lag in population growth response of isochrysis galbana to a variable nitrate environment," Ecology, vol. 50, no. 2, pp. 188-192, 1969.

[38] A. Cunningham and R. M. Nisbet, "Time lag and co-operativity in the transient growth dynamics of microalgae," Journal of Theoretical Biology, vol. 84, no. 2, pp. 189-203, 1980.

[39] Y. Kuang, Delay Differential Equations with Applications in Populatin Dynamics, Academic Press, New York, NY, USA, 1993.

[40] L. Yu, X. Hu, R. Lin, H. Zhang, Z. Nan, and F. Li, "The effects of environmental conditions on the growth of petroleum microbes by microcalorimetry," Thermochimica Acta, vol. 359, no. 2, pp. 95-101, 2000.

[41] X. Mao, Y. Shen, and C. Yuan, "Almost surely asymptotic stability of neutral stochastic differential delay equations with Markovian switching," Stochastic Processes and Their Applications, vol. 118, no. 8, pp. 1385-1406, 2008.

[42] X. Mao, "Stationary distribution of stochastic population systems," Systems \& Control Letters, vol. 60, no. 6, pp. 398-405, 2011.

[43] D. Jiang, N. Shi, and X. Li, "Global stability and stochastic permanence of a non-autonomous logistic equation with random perturbation," Journal of Mathematical Analysis and Applications, vol. 340, no. 1, pp. 588-597, 2008.

[44] D. Jiang, C. Ji, N. Shi, and J. Yu, "The long time behavior of DI SIR epidemic model with stochastic perturbation," Journal of Mathematical Analysis and Applications, vol. 372, no. 1, pp. $162-180,2010$.

[45] M. Liu and K. Wang, "Asymptotic behavior of a stochastic nonautonomous Lotka-Volterra competitive system with impulsive perturbations," Mathematical and Computer Modelling, vol. 57, no. 3-4, pp. 909-925, 2013.

[46] M. Liu and K. Wang, "Global asymptotic stability of a stochastic Lotka-Volterra model with infinite delays," Communications in Nonlinear Science and Numerical Simulation, vol. 17, no. 8, pp. 3115-3123, 2012.

[47] R. Z. KHasminskii, Stochastic Stability of Differential Equations, Sijthoff \& Noordhoff, Alphen aan den Rijn, The Netherlands, 1980.

[48] X. Mao, Stochastic Differential Equations and Their Applications, Horwood Publishing, Chichester, UK, 1997.

[49] V. B. Kolmanovskiǐ and V. R. Nosov, Stability of Functional Differential Equations, Academic Press, New York, NY, USA, 1986.

[50] D. J. Higham, "An algorithmic introduction to numerical simulation of stochastic differential equations," SIAM Review, vol. 43 , no. 3, pp. 525-546, 2001. 


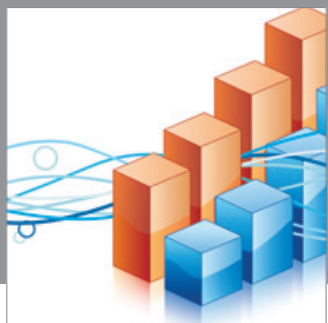

Advances in

Operations Research

mansans

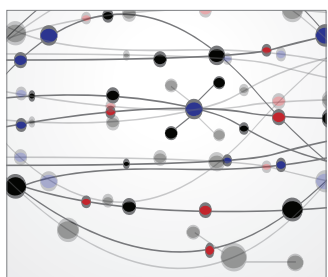

The Scientific World Journal
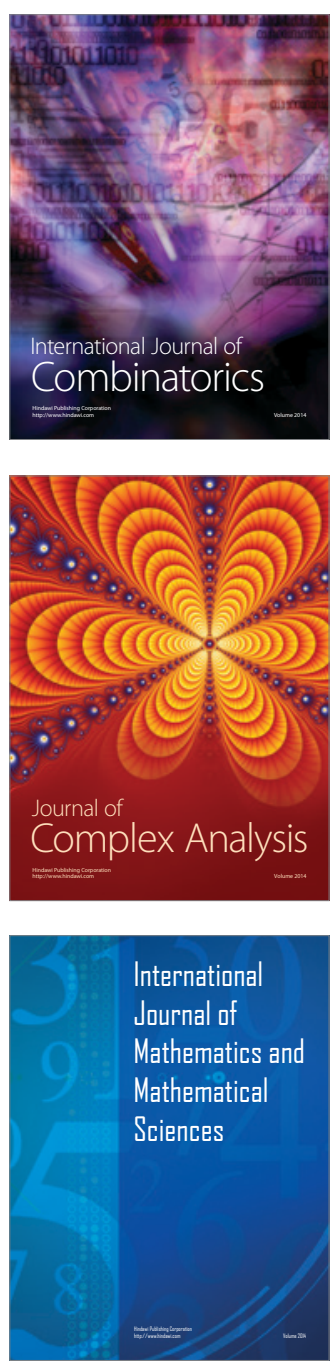
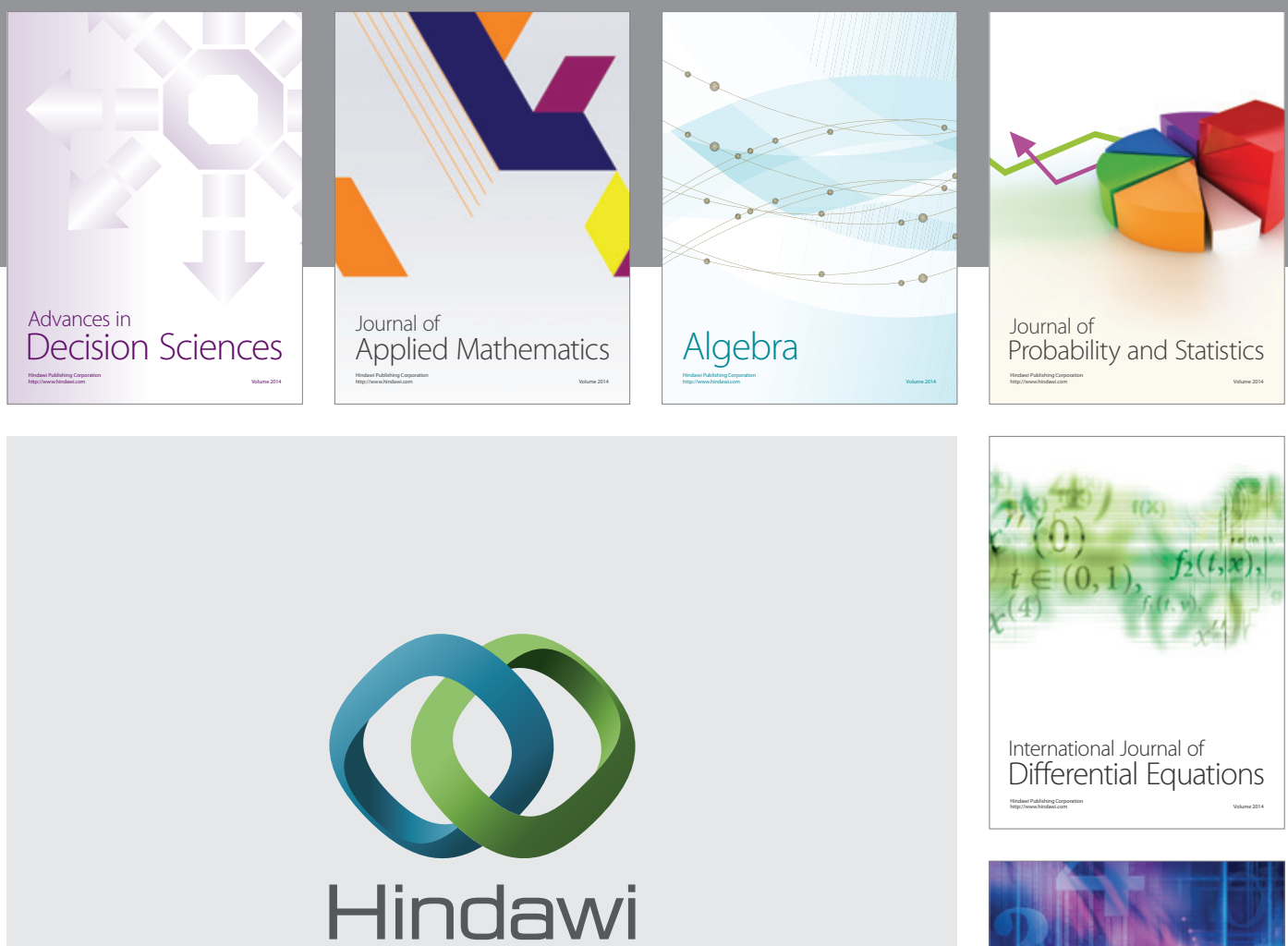

Submit your manuscripts at http://www.hindawi.com
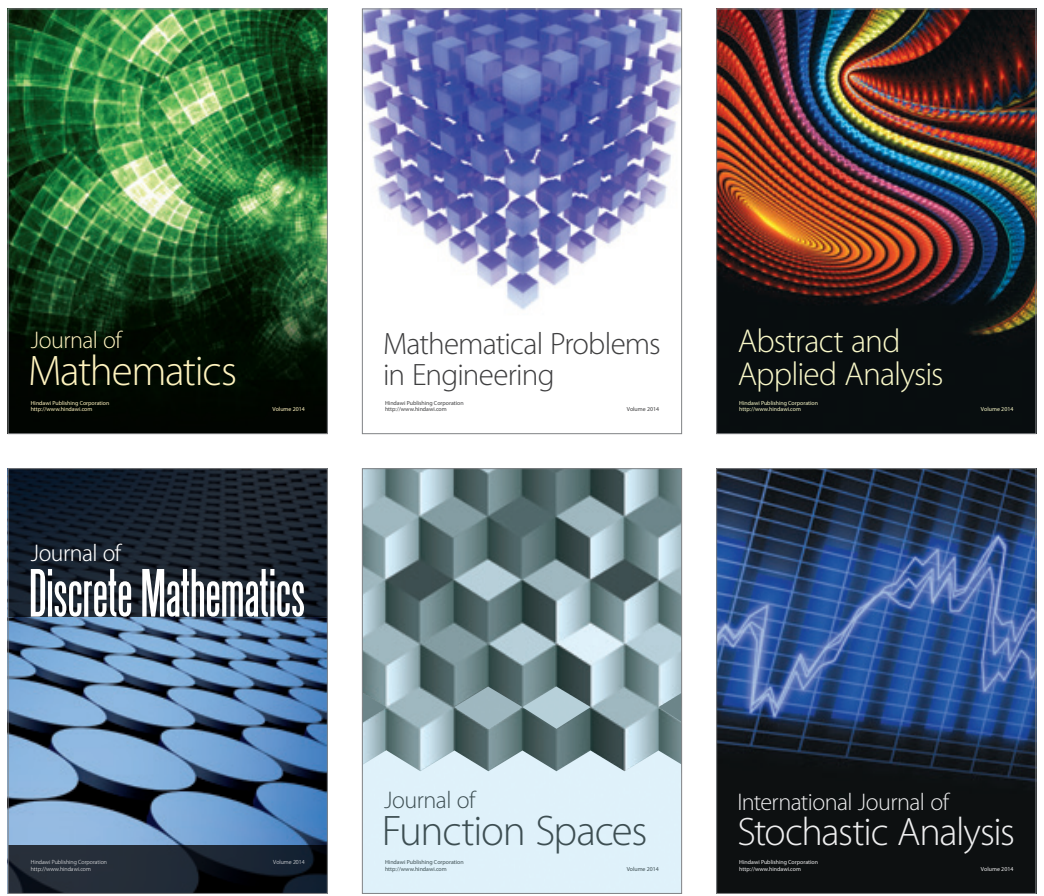

Journal of

Function Spaces

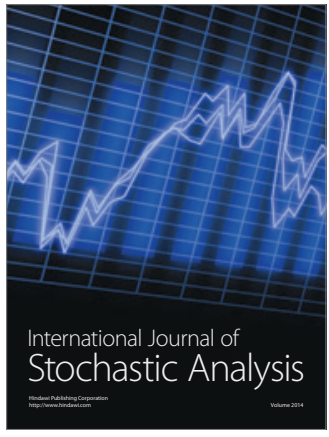

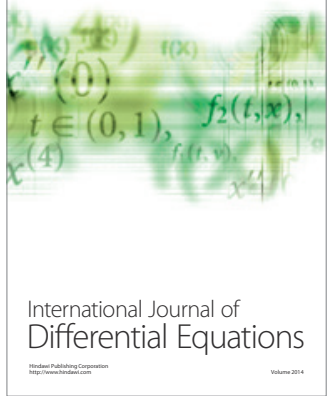
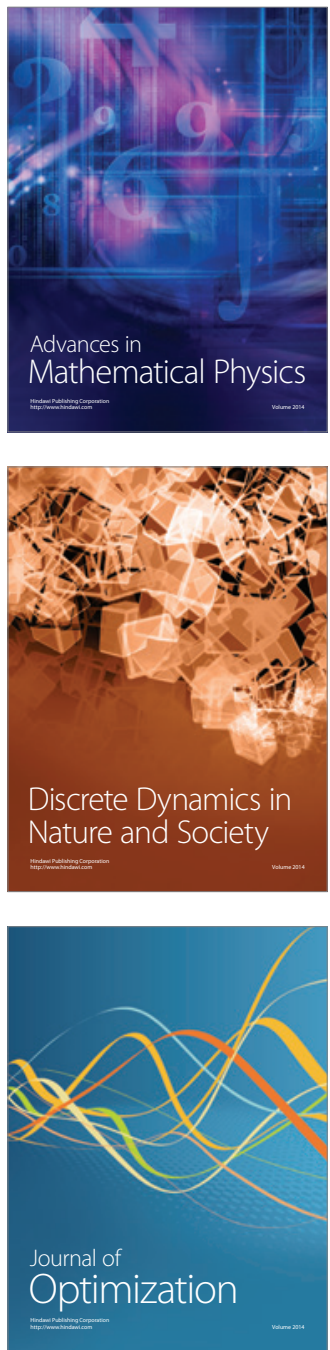